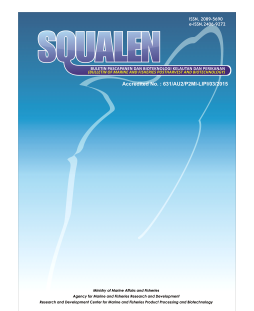

\title{
NON-TARGET SCREENING METHOD FOR THE IDENTIFICATION OF PERSISTENT AND EMERGING ORGANIC CONTAMINANTS IN SEAFOOD AND SEDIMENT FROM JAKARTA BAY
}

\author{
Dwiyitno 1,2,3, Larissa Dsikowitzky², Nuri Andarwulan',4, Hari Eko Irianto5 , Hanifah Nuryani Lioe', \\ Farida Ariyani ${ }^{3}$, Jan Schwarzbauer ${ }^{2}$ \\ 'Department of Food Science and Technology, Bogor Agricultural University. PO Box 220 Bogor 16002 \\ ${ }^{2}$ Institute of Geology and Geochemistry of Petroleum and Coal (GGPC), RWTH Aachen University, \\ Löchnerstraße 4-20, 52056 Aachen, Germany \\ ${ }^{3}$ Research Center and Development for Marine and Fisheries Product Processing and Biotechnology, Jalan KS. Tubun \\ Petamburan VI, Slipi, Central Jakarta 10260, Indonesia \\ ${ }^{4}$ Southeast Asian Food and Agricultural Science and Technology (SEAFAST) Center, \\ JI. Puspa No. 1, Kampus IPB Darmaga, Bogor 16680, Indonesia \\ ${ }^{5}$ Research Center for Fisheries Management and Conservation, Ministry of Marine Affairs and Fisheries, \\ Jl. Pasir Putih II, Ancol Timur, Jakarta 14430, Indonesia \\ Article history: \\ Received: 2 September 2015; Revised: 13 November 2015; Accepted: 28 November 2015
}

\begin{abstract}
Identification of persistent and emerging organic contaminants in green mussels (Perna viridis), various fishes, banana shrimp (Penaeus merguiensis) and sediment samples from Jakarta Bay has been employed. A non-target GC-MS screening approach has identified more than 60 individual organic compounds from the whole fractions either non-polar, semi-polar or acidic-polar compounds. The substances comprised as persistent organic pollutants (POPs) including DDT (dichlorodiphenyl trichloroethane) and its metabolites as well as high molecular weight polycyclic aromatic hydrocarbons (HMW-PAHs). Noteworthy, a number of emerging contaminants detected in the present study have never been reported previously either from the same location or from Asian waters. They include some priority contaminants of non-persistence halogens and emission of technical products, such as di-isopropylnaphthalenes (DIPNs) dichlorobenzene (DCB), dichlorodiphenyl chloroethene (DDMU) and phenylmethoxynaphthalene (PMN). In general, the concentration order of the priority organic contaminants was sediment $>$ green mussel $>$ fishes $>$ shrimp. Further analysis based on the spatial distribution, individual concentrations and bioavailability suggested that some contaminants are applicable as molecular marker for the assessment of anthropogenic emission in Jakarta Bay, i.e. DIPNs, linear alkylbenzenes (LABs), phenylmethoxynaphthalene (PMN), PAHs, dichlorobenzene, DDT and its metabolites.
\end{abstract}

Keywords: non-target screening, persistent organic pollutants, emerging contaminants, seafood, Jakarta Bay

\section{Introduction}

Jakarta Bay is an essential coastal ecosystem as it is situated in the Capital City Jakarta with more than 10 Million inhabitants (BPS, 2014). The bay provides several functions, such as for maritime transportation, supply natural resources (mainly seafood), as recreational facilities, and support waste regulating services. Importantly, the bay is also exposed to high pollution load derived either from up- land regions through discharging rivers into the bay or from direct coastal activities. The terrestrial contamination is estimated to contribute up to $80 \%$ of the pollution in Jakarta Bay (Anon., 2007). Those contaminants are delivered from various sources, such as domestic disposal, industrial waste, transportation, oil spills, etc. (Uneputty \& Evans, 1997). Hence, the burden of the contaminants potentially increases significantly in accordance with the population growth and the development of industrial activities.

${ }^{*}$ Corresponding author.

E-mail: dwiyitno@yahoo.com 
Jakarta city is currently connected to its suburb regions i.e. Bogor, Depok, Tangerang and Bekasi by which the region known as Jabodetabek or Jakarta Metropolitan Area (JMA). There is huge amount of domestic waste polluting Jakarta Bay resulted from 28.3 Million population of JMA. Additionally, more than 20 thousand industries are situated in Jabodetabek and potentially contribute to pollute Jakarta Bay, either from by-products of combustion and solid or liquid disposal. Further on, many hospitals in Jabodetabek are supposed contribute on organic waste disposal, as well as pesticide/herbicide residues from agricultural effluent in suburb regions (Dsikowitzky et al., in draft; Dwiyitno et al., in draft). At least 161 ton/ day of solid waste and 6.5 Million ton/day of liquid waste are discharged into Jakarta Bay (Takarina \& Adiwibowo, 2011; BPLHD, 2014). On a longer period, accumulation of contaminants could damage the aquatic ecosystem and also produce harmful effects on the environment and human health.

Since Jakarta Bay represents an essential ecosystem, in particular regarding fishing and marine/ aquaculture activities for the Jakarta city and surrounding regions, the impact of organic contamination into this bay has to be thoroughly in concern. Studies on the organic pollutants have been reported from Jakarta Bay which was focused on the priority pollutants such as PAHs, DDT, polychlorinated biphenyls (PCBs), tributhyltin (TBT), polybrominated diphenyl ethers (PBDEs) and hexachlorohexanes/ $\mathrm{HCHs}$ (William et al., 2000; Monirith et al., 2003; Sudaryanto et al., 2007). However, all of those studies were conducted based on either single or selected target approach. To date, one of the biggest issues associated with analytical environmental contaminant is the limitation of target analysis which only focuses on selected contaminants. Selected or targeted compound monitoring approach, with mass spectrometry (MS) for example, using selected ion monitoring (SIM) mode would only assess a limited number of analytes, whereas other potentially harmful or non-target analytes might not be detected (Ibanez et al., 2008). For that reason, non-target approach has been implemented to screen a wide range of known or unknown contaminants and identify the transformation products that were not performed in traditional selected analytical method (Hernandez et al., 2012). Non target screening has been showing an alternative approach for the identification of organic contaminants in water and sediment samples in developed countries (Hernandez et al., 2007; Grigoriadou et al., 2008; Schwarzbauer et al., 2010; Schymanski et al., 2014). Different instruments have demonstrated powerful tools for non-target screening analysis, such as LC-TOF-MS (Diaz et al., 2012), LC-
MS (Hird et al., 2014), and GC-MS (Shaikh et al., 2014).

Investigation of organic contaminants in aquatic biota by using non-target screening has not been reported yet. Through bio-magnification pathway via aquatic food web, the contaminants potentially accumulate in the aquatic organism including seafood at which the concentration will increase as the longer of exposure. Notably, studies on the emerging pollutants such as emission of pharmaceutical and personal care products (PPCPs), hormones and other technical/industrial products like DIPNs, phenylmethoxy- naphthalene in Indonesia and Asian regions are very limited or even none (Suzuki et al., 2007; Terasaki et al., 2008; Dsikowitzky et al., 2014). This research was aimed to study the application of non-target screening using GC/MS approach to investigate the presence and distribution of persistent and emerging organic contaminants related to seafood and sediment compartments in Jakarta Bay. The result of this study is important to evaluate contamination profile, determine the priority contaminants, as well as the safety status of seafood from Jakarta Bay.

\section{Material and Methods}

\subsection{Study Site and Sample Collection}

The study was conducted in the coastal system of Jakarta Bay. The bay stretches from 106 $20^{\prime}$ to $107^{\circ} 03^{\prime}$ east longitude, and from $5^{\circ} 10^{\prime}$ to $6^{\circ} 10^{\prime}$ south latitude, and is administratively within three provinces, i.e. Banten, the DKI of Jakarta, and West Java Provinces. The bay receives water from 13 main rivers, some of which flowing through the Jakarta city and 6 main rivers with in the suburb regions. The regions are strongly influenced by typical tropical climate. The northwest monsoon (November-March) brings rainfall into Jakarta metropolitan area, while the Southeast Monsoon (May-September) coincides with the dry season. This nature results in significant variation of precipitation rate between dry and rainy season i.e. $2-118 \mathrm{~mm} / \mathrm{month}$ (dry season) and $158-275 \mathrm{~mm} /$ month during rainy season of 2013 , which was higher than that of $2012(0-79 \mathrm{~mm} / \mathrm{month}$ in dry season and 47$259 \mathrm{~mm} /$ month during rainy season). Unlikely, average temperature in the region was relatively similar during 2012 and 2013 i.e. $27.7-30.8^{\circ} \mathrm{C}$ and $27.3-29.1^{\circ} \mathrm{C}$ respectively (BPLHD Jakarta, 2012; 2013).

A number of 10 different biological samples have been directly collected from local fisherman in Jakarta Bay during October 2012, May and October 2013 in order to represent the dry and rainy season contamination. They include 6 species of demersal and 2 pelagic fishes, 1 shrimp species (banana shrimp/ 


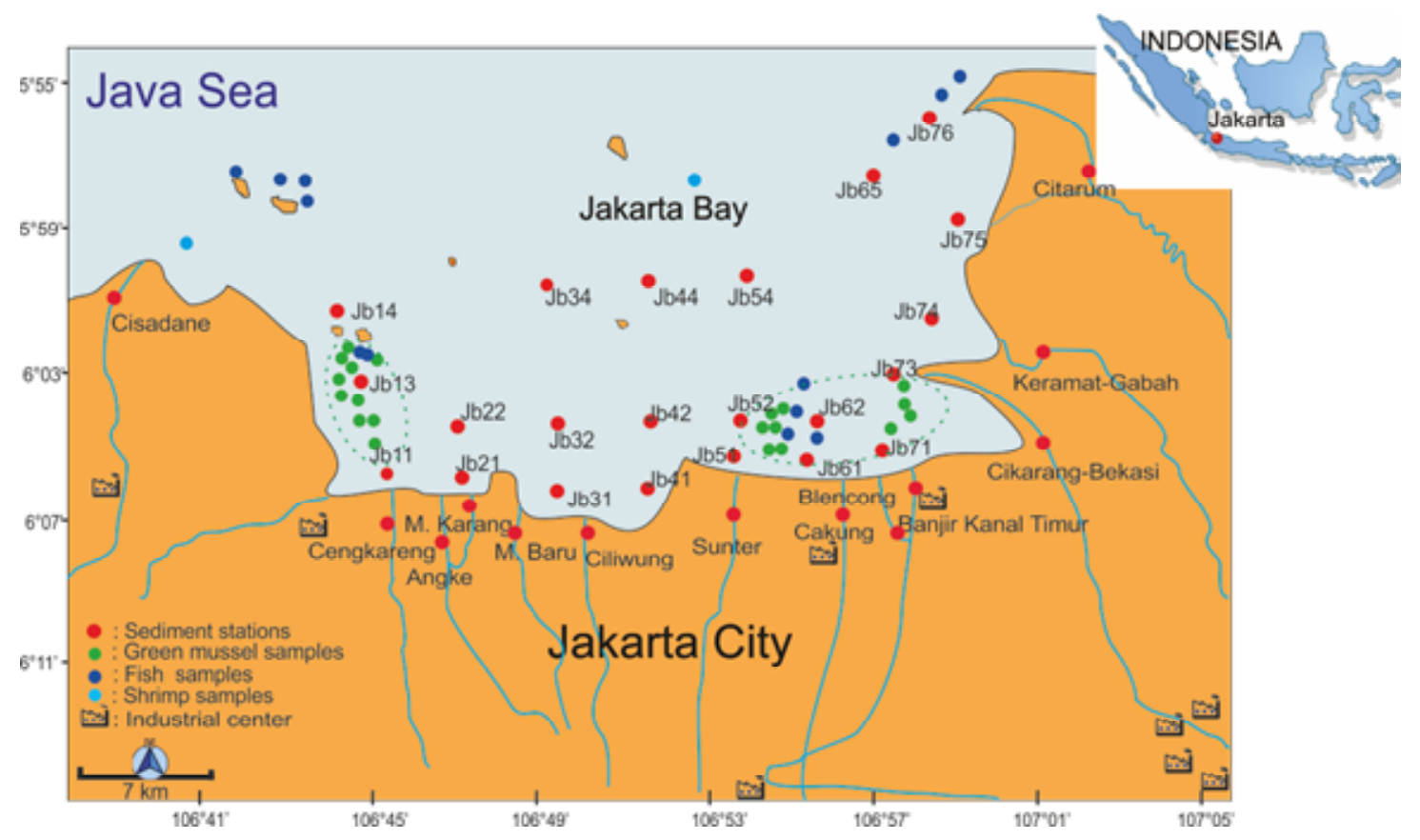

Figure 1.Schematic sketch of the study area and sampling location.

$P$. maerguiensis) as well as green mussel ( $P$. viridis) from 11 different stations. In order to determine contaminant level in the environment compartment, surface sediment was collected from 22 stations in Jakarta Bay with a stainless steel Van Veen Grab $\left(20 \times 20 \mathrm{~cm}^{2}\right)$. During the sampling campaign, all samples were conserved with ice and after reach the laboratory, all samples were stored at $-18^{\circ} \mathrm{C}$ until chemical analysis. Sampling points of biological samples and surface sediment are performed in Figure 1 , while characteristic of biological samples is presented in Table 1.

\subsection{Extraction and Fractionation}

A non-target screening approach was applied based on the method described by Schwarzbauer et al. (2000). Sediment extraction was performed with a high-speed dispersion tool (Ultra-Thurax Turrax ${ }_{25}$ basic, IKA Labortechnich) using mixture of acetone and $n$-hexane, while fractionation was conducted based on the method explained by Franke et al. (2007) into 7 fractions (Figure 2). After separation of the phases, the organic extracts were added $50 \mu \mathrm{l}$ of surrogate standard containing $d 34$-hexadecane $(6.00$ $\mathrm{ng} / \mu \mathrm{l})$, 4-fluoroacetophenone $(7.17 \mathrm{ng} / \mu \mathrm{l})$ and decafluorobenzophenone $(6.96 \mathrm{ng} / \mu \mathrm{l})$. After concentrated, aliquot was dehydrated with sodium sulphate anhydrate $\left(\mathrm{Na}_{2} \mathrm{SO}_{4}\right)$ and eluted with the similar solvent. On the other hand, the polar fraction was further derivatized with diazomethane prior to fractionation as performed in fraction 1-5. Prior to GC and GC/MS-analysis, all extracts were concentrated to approximately $50 \mu \mathrm{l}$ (fraction 1-5) and $\pm 200 \mu \mathrm{l}$ (fraction 6-7). All chemicals were purchased from Sigma-Aldrich, Germany.

Dialysis method using EC-Exsposmeter clean-up semipermeable membrane (ExposMeter sampling technologies, Sweden) was employed for extracting biological samples as described by Dwiyitno et al., in draft. After extracted with dichlorometane (DCM) for 72 hours, supernatant was concentrated and fractioned as performed in sediment sample. One microliter of sample aliquot was injected in to a Trace GC-MS system (ThermoQuest, Germany) linked to HRGC-5160 Mega Series (Carlo Erba, Italy) equipped with a $Z B-X L B$ silica capillary column $(30 \mathrm{~m} \times 0.25 \mathrm{~mm}$ ID, $0.25 \mu \mathrm{m}$ film thickness; Zebron-Phenomenex, Germany). The oven temperature was held at $60^{\circ} \mathrm{C}$ for $3 \mathrm{~min}$ then increased at $3^{\circ} \mathrm{C} / \mathrm{min}$ to $310^{\circ} \mathrm{C}$ and held for $20 \mathrm{~min}$ with injection temperature of $270^{\circ} \mathrm{C}$. Helium was used as carrier gas at $40 \mathrm{~cm} / \mathrm{s}$ velocity. Mass spectrometer was operated in full-scan mode at a resolution of 1000 with a source temperature of $200^{\circ} \mathrm{C}$. Electron impact ionization mode $\left(\mathrm{El}^{+}, 70 \mathrm{eV}\right)$ was operated at a scanning mode from 35 to $700 \mathrm{amu}$ at a rate of $1 \mathrm{~s} / \mathrm{scan}$. The schematic flow of the analysis method is summarized in Figure 2.

\subsection{Identification of organic contaminants}

Individual compound was identified by comparison of the El-Mass spectra and specific ion fragment (Table 2) with those of mass spectral libraries (NIST98 
Table 1. Characteristic of investigated fish, shrimp and mussel species from Jakarta Bay

\begin{tabular}{|c|c|c|c|c|c|}
\hline No & $\begin{array}{l}\text { Common name/ } \\
\text { Scientific name/ } \\
\text { Local name }\end{array}$ & $\begin{array}{l}\text { Habitat/ } \\
\text { Feeding } \\
\text { habit }\end{array}$ & $\begin{array}{l}\text { Average of } \\
\text { weight }+ \text { S.D. } \\
\text { (g) }\end{array}$ & $\begin{array}{l}\text { Average of } \\
\text { length }+ \text { S. } D . \\
(\mathrm{cm})\end{array}$ & $\begin{array}{l}\text { Sampling } \\
\text { period }\end{array}$ \\
\hline 1 & $\begin{array}{l}\text { Green mussel/ } \\
\text { Perna viridis/ } \\
\text { Kerang hijau }\end{array}$ & $\begin{array}{c}\text { Pillar/ } \\
\text { filter feeder }\end{array}$ & $\begin{array}{c}16.0 \pm 6.5 \\
12.4 \pm 2.1 \\
16.4 \pm 6.4\end{array}$ & $\begin{array}{c}6.8 \pm 0.8 \\
6.3 \pm 1.2 \\
7.0 \pm 1.0\end{array}$ & \multirow{2}{*}{$\begin{array}{c}\text { October 2012, } \\
\text { May 2013, } \\
\text { October } 2013\end{array}$} \\
\hline 2 & $\begin{array}{l}\text { Mackerel/ } \\
\text { Rastrelliger kanagurta/ } \\
\text { Kembung }\end{array}$ & $\begin{array}{l}\text { Pelagic/ } \\
\text { omnivore }\end{array}$ & $\begin{array}{l}149.4 \pm 13.1 \\
87.2 \pm 12.8 \\
88.5 \pm 20.0\end{array}$ & $\begin{array}{c}20.7 \pm 0.6 \\
16.3 \pm 0.75 \\
16.3 \pm 1.1\end{array}$ & \\
\hline 3 & $\begin{array}{l}\text { Slender shad/ } \\
\text { Ilisha elongata/ } \\
\text { Mata belo }\end{array}$ & $\begin{array}{l}\text { Pelagic/ } \\
\text { carnivore }\end{array}$ & $103.2 \pm 15.2$ & $113.5 \pm 7.1$ & \multirow{3}{*}{ October 2012} \\
\hline 4 & $\begin{array}{l}\text { White emperor/ } \\
\text { Lethrinus lentjan/ } \\
\text { Lencam }\end{array}$ & $\begin{array}{l}\text { Demersal/ } \\
\text { carnivore }\end{array}$ & $108.3 \pm 30.4$ & $15.7 \pm 1.9$ & \\
\hline 5 & $\begin{array}{l}\text { Streaked spinefoot/ } \\
\text { Siganus javus/ } \\
\text { Baronang }\end{array}$ & $\begin{array}{l}\text { Demersal/ } \\
\text { herbivore }\end{array}$ & $183.1 \pm 32.3$ & $16.8 \pm 1.0$ & \\
\hline 6 & $\begin{array}{l}\text { Johnii snapper/ } \\
\text { Lutjanus johnii/ } \\
\text { lkan tanda }\end{array}$ & $\begin{array}{l}\text { Demersal/ } \\
\text { carnivore }\end{array}$ & $\begin{array}{l}103.2 \pm 15.2 \\
118.5 \pm 33.4\end{array}$ & $\begin{array}{l}14.4 \pm 0.9 \\
15.6 \pm 0.9\end{array}$ & \multirow{3}{*}{$\begin{array}{c}\text { October } 2012, \\
\text { May } 2013\end{array}$} \\
\hline 7 & $\begin{array}{l}\text { Croaker/ } \\
\text { Argyrosomus amoyensis/ } \\
\text { Gulamah }\end{array}$ & $\begin{array}{l}\text { Demersal/ } \\
\text { omnivore }\end{array}$ & $\begin{array}{l}204.6 \pm 37.3 \\
183.9 \pm 58.9\end{array}$ & $\begin{array}{l}21.0 \pm 1.2 \\
21.1 \pm 2.1\end{array}$ & \\
\hline 8 & $\begin{array}{l}\text { Banana shrimp/ } \\
\text { Penaeus maerguiensis/ } \\
\text { Udang putih }\end{array}$ & $\begin{array}{l}\text { Demersal/ } \\
\text { omnivore }\end{array}$ & $\begin{array}{l}26.2 \pm 6.2 \\
25.1 \pm 7.9\end{array}$ & $\begin{array}{l}13.8 \pm 2.6 \\
12.8 \pm 2.8\end{array}$ & \\
\hline 9 & $\begin{array}{l}\text { White-spotted rabbitfish/ } \\
\text { Siganus canaliculatus/ } \\
\text { Baronang susu }\end{array}$ & $\begin{array}{l}\text { Demersal/ } \\
\text { herbivore }\end{array}$ & $\begin{array}{c}62.1 \pm 7.2 \\
91.7 \pm 15.8\end{array}$ & $\begin{array}{l}12.8 \pm 0.7 \\
14.4 \pm 1.3\end{array}$ & \multirow{2}{*}{$\begin{array}{c}\text { May } 2013, \\
\text { October } 2013\end{array}$} \\
\hline 10 & $\begin{array}{l}\text { Sea catfish/ } \\
\text { Netuma thalassina/ } \\
\text { Manyung }\end{array}$ & $\begin{array}{l}\text { Demersal/ } \\
\text { carnivore }\end{array}$ & $\begin{array}{c}467.9 \pm 67.8 \\
588.6 \pm 246.4\end{array}$ & $\begin{array}{l}29.9 \pm 1.7 \\
33.4 \pm 5.5\end{array}$ & \\
\hline
\end{tabular}

"Fish length is measured as standard length

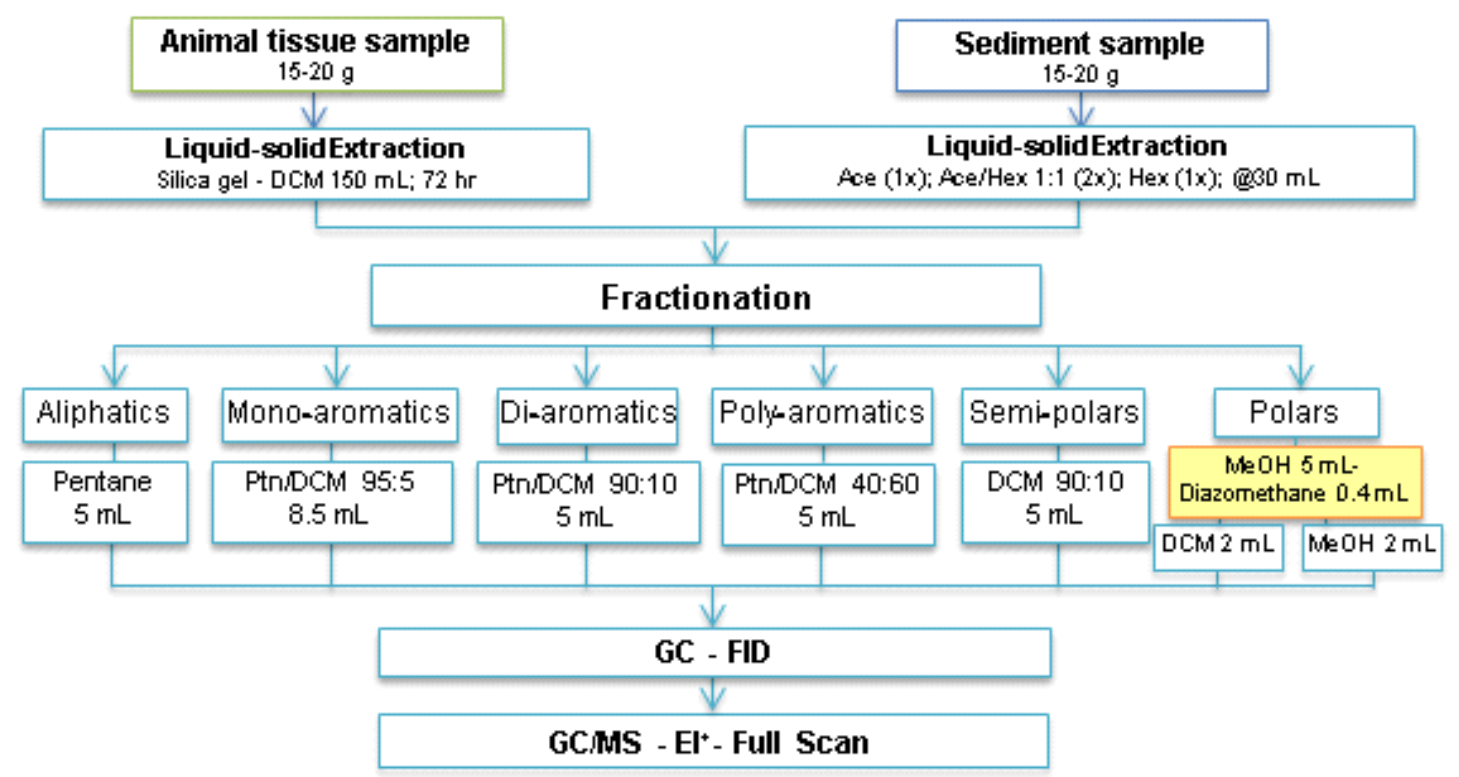

Figure 2. Schematic flow of non-target screening approach (Schwarzbauer et al., 2010; Dwiyitno et al., in draft) (DCM: dichloromethane; Ace: Acetone; Hex: Hexane; Ptn: Pentane; MeOH: Methanol). 
and Wiley $4^{\text {th }}$ Ed.), as well as by integrating the gas chromatographic retention time or scan number of authentic reference materials. Quantitation was performed by integrating ion chromatograms on selected $\mathrm{m} / \mathrm{z}$ and calculated by Xcalibur based on the comparison of sample spectral and response factors (RF) of reference materials. Contaminant concentration was calculated as follow:

Concentration $\left[\frac{\mathrm{ng}}{\mathrm{g}}\right]=\frac{\text { Area of sample }}{\text { Area of surrogate }} \times$ Response $\times \frac{\text { Surrogate standard }(\mathrm{ng})}{\text { factor }}$ standard

\subsection{Determination of Dry Weight and Total Organic Matter}

Organic contaminant concentrations in the animal tissue samples were given based on fat content, while the contaminant concentrations in sediment samples were presented based on total organic matter content. Dry weights were determined by drying of $\pm 2 \mathrm{~g}$ sample aliquots at $105^{\circ} \mathrm{C}$ for 18 hours to constant weight (Heiri et al., 2001). Total organic matter contents were further calculated by determining the loss of ignition (LOI) at $550^{\circ} \mathrm{C}$ using Nabertherm furnace (LT 15/13) for 3 hours.

\subsection{Determination of fat content}

Total fat content was quantified based on gravimetric method using soxhlet system (AOAC, 2002). Approximately $2.0 \mathrm{~g}$ of fresh seafood tissue was weighed and placed in an extraction thimble and placed in the soxhlet apparatus. Fat was extracted through repeated washing/percolation with $150 \mathrm{~mL}$ petroleum ether for \pm 6 hours. After extraction, the fat extract collected in the extraction pot was oven dried and weighed in order to compare to the weight of the initial sample.

\section{Result and Discussion}

Non target GC/MS screening analysis applied on green mussels, fish species, shrimp, and sediment samples of Jakarta Bay revealed a wide range accumulation of lipophilic organic substances. Table 2 represents the summary of more than 60 individual organic contaminants identified from the whole fractions, whereas concentrations of selected priority compounds are given in Table 3 . In general, sediment samples accumulate more various with the higher concentration of organic contaminants in comparison to biological samples (green mussel, fishes and shrimp).

The solid-liquid extraction method on biological samples and sediment produced seven fractions representing the non-polar, semi-polar and acidic relatively polar compounds respectively. However, the majority of contaminants were detected in fractions 2-5, while minor contaminants were also identified in fraction 1, 6 and 7 . This indicates that the majority of organic contaminants were semi polar substances, such as halogenated compounds (fractions 2-4), aromatic compounds and technical products (fractions 2-5), as presented in Figure 2. Fraction 1 consisted of relatively non-polar compounds, while methylated of relatively polar compounds were eluted in fractions 6 and 7 . In contrast, low volatile polar compounds, such as organotins, are typically more sensitively and selectively detected using liquid chromatography instead of gas chromatography (Zou \& Schreiber, 2012).

Spatial distribution showed that contaminant concentration in green mussel from eastern part of Jakarta Bay was relatively higher than that from the western part. This difference seems correspond to the contaminant concentration in the environment compartment as presented in sediment sample (Figure 4-7). Additionally, contaminant concentration in river sediment was higher than that of coastal sediment, indicating the contaminant origin were predominantly from terrestrial emission. This fact was also supported the fact that contaminant concentration in coastal sediment apparently reflected the river sediment concentration.

Due to hydrophobic property, lipophilic compounds tend to be separated from the aqueous phase for the further adsorption into colloidal material or organic matter or sediment by van der Waals force (Walker, 1987). Hydrophobicity of organic compound is commonly indicated by coefficient partition of octanolwater $\left(\mathrm{K}_{\text {ow }}\right)$. Persistency of organic compounds in aquatic ecosystem is also affected by their chemical stability to the chemical degradation such as photochemical and microbial degradation (Ricking \& Schwarzbauer, 2012). Environmental behavior like $\mathrm{pH}$, temperature, oxygen availability may also contribute in the nature of contaminant accumulation (Kim et al., 2003).

The relatively less variety and lower concentration of organic contaminants in fish and shrimp species compared to that of green mussel in the present study suggested the different uptake and metabolism nature, as explained earlier by Dwiyitno et al. (in draft). Earlier studies showed that occurrence of elimination rate of xenobiotic in mussel species tends to be lower than that of fish species (D'Adamo et al., 1997; Zhao et al., 2014). With regard to shrimp species, relatively low detoxification rate was performed on the bioaccumulation study of PCBs on Palaemonetes 
Table 2. Organic compounds identified in sediment and biological samples of Jakarta Bay

\begin{tabular}{|c|c|c|c|c|c|c|c|}
\hline No & Organic Compound & Ion Fragment & $\begin{array}{l}\text { Scan Nr. } \\
\text { (c.a.) }\end{array}$ & $\begin{array}{l}\text { Green } \\
\text { mussels }\end{array}$ & Fishes & Shrimp & Sediment \\
\hline A & HALOGENATED COMPOUNDS & & & 3 & 5 & 1 & 7 \\
\hline 1 & $\begin{array}{l}\text { Bis(4-chlorophenyl)-1,1,1- } \\
\text { trichloroethane (DDT) }\end{array}$ & $235,237,354$ & $4860-5175$ & - & $\sqrt{ }$ & - & $\sqrt{ }$ \\
\hline 2 & $\begin{array}{l}\text { Bis (4-chlorophenyl)-1,1- } \\
\text { dichloroethane (DDD) }\end{array}$ & $235,237,320$ & $3860-4744$ & - & $\sqrt{ }$ & - & - \\
\hline 3 & $\begin{array}{l}\text { Bis (4-chlorophenyl)-1,1- } \\
\text { dichloroethene (DDE) }\end{array}$ & $246,248,318$ & $4450-4690$ & $\sqrt{ }$ & $\sqrt{ }$ & $\sqrt{ }$ & $\sqrt{ }$ \\
\hline 4 & $\begin{array}{l}\text { Bis (4-chlorophenyl)-1- } \\
\text { chloroethylene (DDMU) }\end{array}$ & $212,282,284$ & 4510 & $\sqrt{ }$ & $\sqrt{ }$ & - & $\sqrt{ }$ \\
\hline 5 & Dichlorobenzenes & 146,148 & 370 & $\sqrt{ }$ & $\sqrt{ }$ & - & $\sqrt{ }$ \\
\hline 6 & Trichloromethylbenzyl-acetate & 149,107 & 2570 & - & - & - & $\sqrt{ }$ \\
\hline 7 & Methyltriclosan & 304,254 & 4420 & - & - & - & $\sqrt{ }$ \\
\hline 8 & Chlorpyrifos & 316,199 & 4100 & - & - & - & $\sqrt{ }$ \\
\hline B & $\begin{array}{l}\text { POLYCYCLIC AROMATIC } \\
\text { HYDROCARBONS (PAHs) }\end{array}$ & & & 27 & 19 & 5 & 33 \\
\hline 9 & Naphthalene & 128 & 1000 & $\sqrt{ }$ & $\sqrt{ }$ & $\sqrt{ }$ & $\sqrt{ }$ \\
\hline 10 & C1 to C4-Naphthalenes & $\begin{array}{r}142,156,170 \\
184\end{array}$ & $1510-3300$ & $\sqrt{ }$ & $\sqrt{ }$ & $\sqrt{ }$ & $\sqrt{ }$ \\
\hline 11 & Biphenyl & 154,76 & $1880-1920$ & $\sqrt{ }$ & $\sqrt{ }$ & & $\sqrt{ }$ \\
\hline 12 & C1 to C4-Biphenyls & $\begin{array}{r}168,182,196 \\
210\end{array}$ & $2050-3200$ & $\sqrt{ }$ & $\sqrt{ }$ & $\sqrt{ }$ & $\sqrt{ }$ \\
\hline 13 & Acenaphtylene & 152,176 & 2180 & $\sqrt{ }$ & $\sqrt{ }$ & - & $\sqrt{ }$ \\
\hline 14 & Acenaphthene & 154,153 & 2270 & $\sqrt{ }$ & $\sqrt{ }$ & - & $\sqrt{ }$ \\
\hline 15 & Fluorene & 166,165 & $1690-2770$ & $\sqrt{ }$ & $\sqrt{ }$ & - & $\sqrt{ }$ \\
\hline 16 & $\mathrm{C} 1$ and $\mathrm{C} 2$-Fluorenes & 180,194 & $3080-3530$ & $\sqrt{ }$ & $\sqrt{ }$ & - & $\sqrt{ }$ \\
\hline 17 & Phenanthrene & 178 & 3350 & $\sqrt{ }$ & $\sqrt{ }$ & $\sqrt{ }$ & $\sqrt{ }$ \\
\hline 18 & Anthracene & 178 & 3450 & $\sqrt{ }$ & $\sqrt{ }$ & $\sqrt{ }$ & $\sqrt{ }$ \\
\hline 19 & $\begin{array}{l}\text { C1 and C2-Anthracene/- } \\
\text { Phenanthrene }\end{array}$ & 192,206 & $3680-4100$ & $\sqrt{ }$ & $\sqrt{ }$ & - & $\sqrt{ }$ \\
\hline 20 & Fluoranthene & 202 & 4200 & $\sqrt{ }$ & $\sqrt{ }$ & - & $\sqrt{ }$ \\
\hline 21 & Pyrene & 202 & 4460 & $\sqrt{ }$ & $\sqrt{ }$ & - & $\sqrt{ }$ \\
\hline 22 & C1- Fluoranthene/Pyrene & 216,215 & $4740-4890$ & $\sqrt{ }$ & - & - & $\sqrt{ }$ \\
\hline 23 & $\begin{array}{l}\text { Chrysene/Benz(a)anthracene/ } \\
\text { Triphenylene }\end{array}$ & 228 & $5320-5420$ & $\sqrt{ }$ & - & - & $\sqrt{ }$ \\
\hline 24 & C1-Chrysene/Benz(a)anthraceı & 242 & 4590 & $\sqrt{ }$ & - & - & $\sqrt{ }$ \\
\hline 25 & $\begin{array}{l}\text { Benzaceanthrylene/- } \\
\text { acephenanthrylene/- } \\
\text { fluoranthene }\end{array}$ & 252 & 5570 & $\sqrt{ }$ & - & - & $\sqrt{ }$ \\
\hline 26 & $\begin{array}{l}\text { Benz(a)pyrene/Benz(e)pyrene/ } \\
\text { Benz(k)fluoranthene }\end{array}$ & 252 & 6490 & $\sqrt{ }$ & - & - & $\sqrt{ }$ \\
\hline 27 & Perylene & 252 & 6580 & $\sqrt{ }$ & - & - & $\sqrt{ }$ \\
\hline 28 & Dibenzo(a)Anthracene & 278 & 7100 & - & - & - & $\sqrt{ }$ \\
\hline 29 & Indeno(1,2,3-cd)pyrene & 276,138 & 7240 & - & - & - & $\sqrt{ }$ \\
\hline 30 & Benzo(ghi)perylene & 276,138 & 7250 & - & - & - & $\sqrt{ }$ \\
\hline
\end{tabular}


Table 2. Organic compounds (continued)

\begin{tabular}{|c|c|c|c|c|c|c|c|}
\hline No & Organic Compound & Ion Fragment & $\begin{array}{l}\text { Scan Nr. } \\
\text { (c.a.) }\end{array}$ & $\begin{array}{c}\text { Green } \\
\text { mussels }\end{array}$ & Fishes & Shrimp & Sediment \\
\hline 31 & Tetraline & 132,104 & 920 & $\sqrt{ }$ & - & - & $\sqrt{ }$ \\
\hline 32 & $\begin{array}{l}\text { C1 to } \mathrm{C} 3 \text {-Tetralines or } \mathrm{C} 1 \\
\text { to } \mathrm{C} 4 \text {-Indanes }\end{array}$ & $\begin{array}{r}146,160,174 \\
188,202\end{array}$ & $1500-2400$ & $\sqrt{ }$ & $\sqrt{ }$ & & $\sqrt{ }$ \\
\hline 33 & Decaline & 138,109 & 520 & - & - & - & $\sqrt{ }$ \\
\hline 34 & $\begin{array}{l}\text { C5-Decalines or C6- } \\
\text { Octahydroindane }\end{array}$ & $166-208$ & $1970-2045$ & & $\sqrt{ }$ & - & $\sqrt{ }$ \\
\hline 35 & Tetrahydroreten (THR) & 238,223 & 4290 & $\sqrt{ }$ & - & - & $\sqrt{ }$ \\
\hline 36 & Dibenzothiophene & 184,139 & 3280 & $\sqrt{ }$ & $\sqrt{ }$ & & $\sqrt{ }$ \\
\hline 37 & $\begin{array}{l}\text { C1 and C2- } \\
\text { Dibenzothiophene }\end{array}$ & 198,212 & $3530-3660$ & $\sqrt{ }$ & $\sqrt{ }$ & & $\sqrt{ }$ \\
\hline 38 & C1-Dihydronaphthalene & 172,157 & $1760-1830$ & - & - & - & $\sqrt{ }$ \\
\hline 39 & Dibenzofuran & 169,139 & 2490 & $\sqrt{ }$ & $\sqrt{ }$ & - & $\sqrt{ }$ \\
\hline 40 & C1 and C2-Dibenzofurans & 182,181 & 2880 & $\sqrt{ }$ & $\sqrt{ }$ & - & $\sqrt{ }$ \\
\hline 41 & $\begin{array}{l}\text { Hydroxy-3-methyl-1,4- } \\
\text { naphtoquinone }\end{array}$ & 188,131 & $2800-2940$ & - & - & - & $\sqrt{ }$ \\
\hline $\mathbf{C}$ & ALKYLBENZENES & & & 2 & 2 & 2 & 2 \\
\hline 42 & C2 to C6-Benzenes & $\begin{array}{r}106,120,134 \\
148,162\end{array}$ & $10-940$ & $\sqrt{ }$ & $\sqrt{ }$ & $\sqrt{ }$ & $\sqrt{ }$ \\
\hline 43 & $\begin{array}{l}\text { Linear alkylbenzenes }\left(\mathrm{C}_{10}\right. \\
\text { to } \mathrm{C}_{13} \text { - } \mathrm{LABs} \text { ) }\end{array}$ & $\begin{array}{r}218,232,246 \\
260 \\
\end{array}$ & $2320-3500$ & $\sqrt{ }$ & $\sqrt{ }$ & $\sqrt{ }$ & $\sqrt{ }$ \\
\hline $\mathrm{D}$ & STEROIDS & & & - & - & - & 2 \\
\hline 44 & Coprostanol & 388 & 6540 & - & - & - & $\sqrt{ }$ \\
\hline 45 & Cholesterol & 386 & 6840 & - & - & - & $\sqrt{ }$ \\
\hline $\bar{E}$ & ISOPRENOIDS & & & 2 & 2 & - & 2 \\
\hline 46 & Limonene & 136,93 & 410 & $\sqrt{ }$ & $\sqrt{ }$ & - & $\sqrt{ }$ \\
\hline 47 & Cadalene & 198,183 & 3000 & $\sqrt{ }$ & $\sqrt{ }$ & - & $\sqrt{ }$ \\
\hline $\mathbf{F}$ & TECHNICAL PRODUCTS & & & 9 & 3 & 3 & 15 \\
\hline 48 & $\begin{array}{l}\text { Di-iso-propylnaphthalenes } \\
\text { (DIPNs) }\end{array}$ & 212,197 & $2880-3200$ & $\sqrt{ }$ & $\sqrt{ }$ & $\sqrt{ }$ & $\sqrt{ }$ \\
\hline 49 & Phenylmethoxynaphthalen & 234,91 & 4500 & $\sqrt{ }$ & $\sqrt{ }$ & $\sqrt{ }$ & $\sqrt{ }$ \\
\hline 50 & $\begin{array}{l}\mathrm{N}, \mathrm{N},- \text {-Diethyl- } m \text {-toluamide } \\
\text { (DEET) }\end{array}$ & 191,119 & 2590 & - & - & - & $\sqrt{ }$ \\
\hline 51 & Tributylphosphate & 99 & 2860 & $\sqrt{ }$ & - & - & $\sqrt{ }$ \\
\hline 52 & Phenyl-terphenyl & 306 & $6560-6820$ & $\sqrt{ }$ & - & - & $\sqrt{ }$ \\
\hline 53 & Terphenyl & 230 & $3750-6820$ & $\sqrt{ }$ & - & - & $\sqrt{ }$ \\
\hline 54 & Phenyl naphthalene & 203,179 & 3690 & $\sqrt{ }$ & - & - & - \\
\hline 55 & Aminoacetophenone & 135,12 & 1750 & - & - & - & $\sqrt{ }$ \\
\hline 56 & Trichloroethylphenyl & 149,107 & 2570 & - & - & - & $\sqrt{ }$ \\
\hline 57 & Formado acetophenone & 163,135 & 2750 & - & - & - & $\sqrt{ }$ \\
\hline 59 & Trimethyl-1,3-pentandi- & 71 & 2620 & $\sqrt{ }$ & - & - & $\sqrt{ }$ \\
\hline 60 & Diphenylether & 170,141 & 1980 & - & - & - & $\sqrt{ }$ \\
\hline 61 & Isopropylbiphenyl & 200 & 3470 & $\sqrt{ }$ & - & - & - \\
\hline 62 & Diisopropylbiphenyl & 238,223 & 3870 & - & - & - & $\sqrt{ }$ \\
\hline 63 & Phenyl trimethylindane & 236,221 & $3110-3200$ & $\sqrt{ }$ & $\sqrt{ }$ & $\sqrt{ }$ & $\sqrt{ }$ \\
\hline \multirow[t]{2}{*}{64} & Indole & 90,117 & 1690 & - & - & - & $\sqrt{ }$ \\
\hline & Total & & & 44 & 31 & 11 & 59 \\
\hline
\end{tabular}

$\checkmark$ : detectable; - : non detectable (LOQ: $0.5 \mathrm{ng}$ ) 


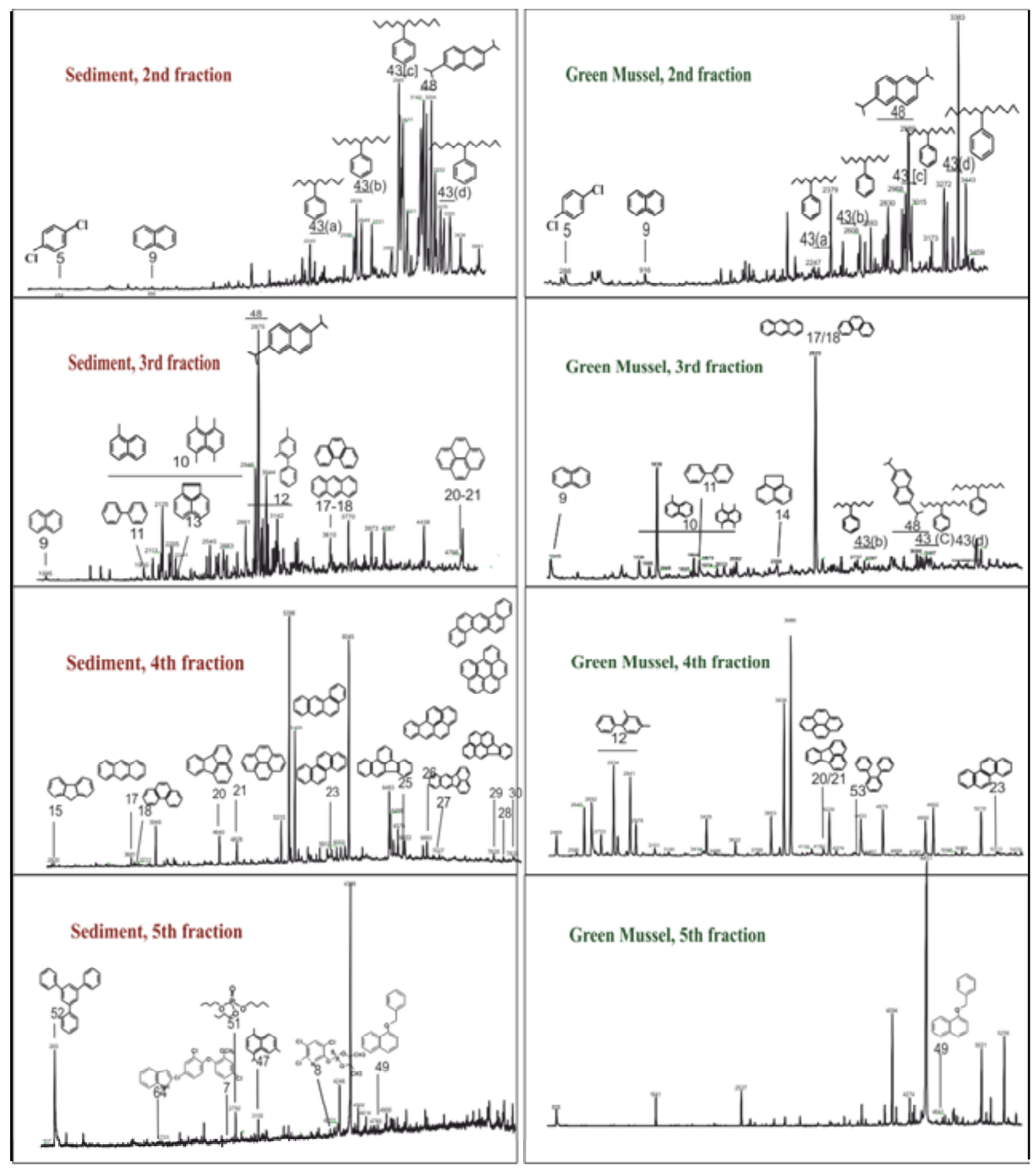

Figure 3. Comparison of total ion chromatogram (TIC) of different fractions between sediment and green mussel samples (numbers of identified compounds correspond to the sample numbers in Table 2).

varians shrimp conducted by Grilo et al. (2014). Noteworthy, concentration of contaminants in the environment is crucial factor affecting their bioaccumulation into aquatic animal. In Jakarta Bay, green mussel is cultivated in the relatively contaminated environment near the coast of western (Dadap and Kamal) and eastern part (Muara Tawar and Cilincing) of the bay (Figure 1).
Basically, aquatic animal accumulates xenobiotics through different routes i.e. direct uptake from water phase via epidermis organs or gill (bioconcentration) and via gastrointestinal tract from ingestion of either suspended particulate matter or from consumption of contaminated feed or known as bioaccumulation and biomagnification (Pierard et al., 1996). Further on, bioavailability of the contaminant in the environment 

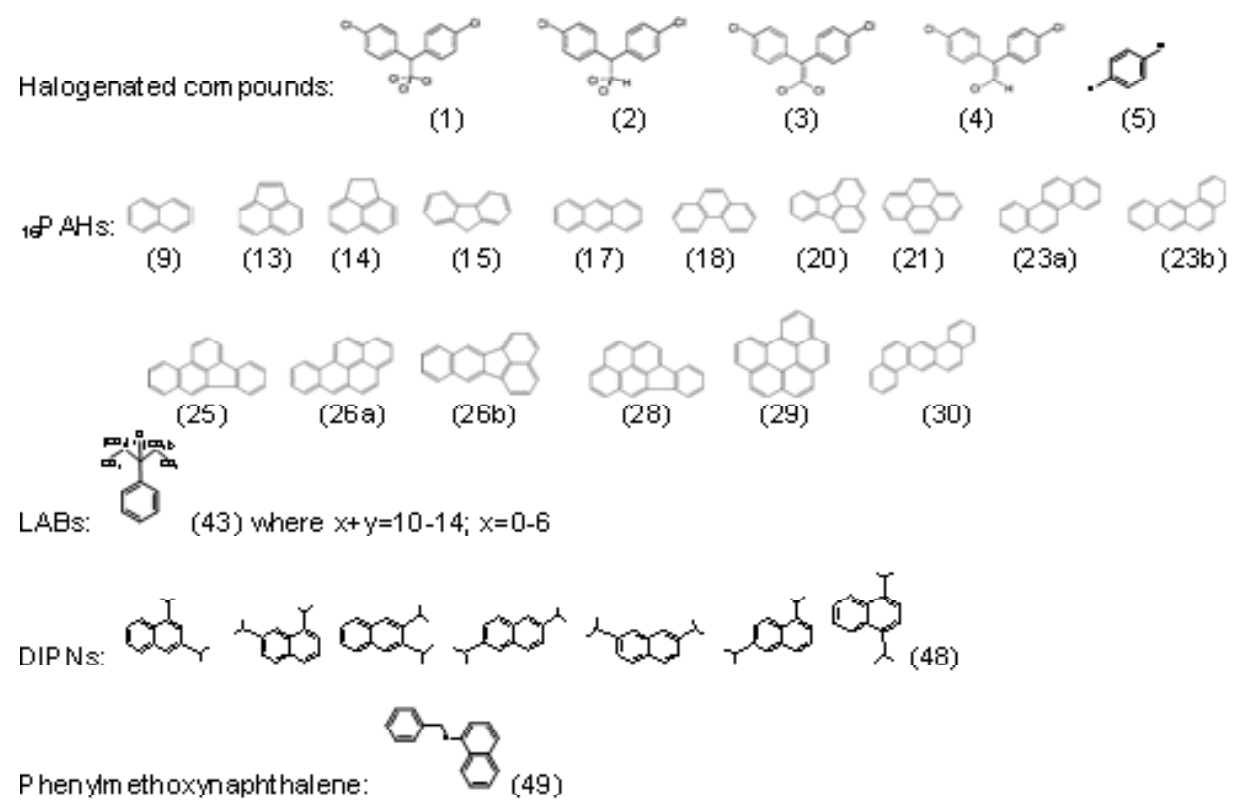

Figure 3. Chemical structures of important contaminants identified in sediment, green mussel and fish samples (the numbers correspond to the sample numbers in Table 2)

Table 3. Geometric mean and (min-max) concentration of priority contaminants in Jakarta Bay

\begin{tabular}{|c|c|c|c|c|c|c|c|}
\hline \multirow[b]{2}{*}{ No } & \multirow{2}{*}{$\begin{array}{l}\text { Organic } \\
\text { Compound }\end{array}$} & \multicolumn{2}{|c|}{ Mussels (ng/g Fat) } & \multirow{2}{*}{$\begin{array}{c}\text { Fish } \\
\text { (ng/g Fat) }\end{array}$} & \multirow{2}{*}{$\begin{array}{l}\text { Shrimp } \\
\text { (ng/g Fat) }\end{array}$} & \multirow{2}{*}{$\begin{array}{c}\text { Jakarta Bay } \\
\text { Sediment } \\
\text { (ng/g TOC) }\end{array}$} & \multirow{2}{*}{$\begin{array}{c}\text { River } \\
\text { Sediment } \\
\text { (ng/g TOC) }\end{array}$} \\
\hline & & West & East & & & & \\
\hline 1 & $\begin{array}{l}\text { Dichlorodiphenyl- } \\
\text { trichloretane (DDX) }\end{array}$ & $\begin{array}{r}90 \\
\text { (n.d.-220) }\end{array}$ & $80^{*}$ & $\begin{array}{r}90 \\
\text { (n.d. }-470)\end{array}$ & $\left.75^{*}\right)$ & $\begin{array}{r}30 \\
\text { (n.d.-80) }\end{array}$ & $\begin{array}{r}1,04 \\
\text { (n.d.-1,560) }\end{array}$ \\
\hline 2 & $\begin{array}{l}\text { Dichlorobenzene } \\
\text { (DCB) }\end{array}$ & $\begin{array}{r}280 \\
(110-950)\end{array}$ & $\begin{array}{r}280 \\
(\text { n.d.-1,100) }\end{array}$ & $\begin{array}{r}40 \\
(\text { n.d. }-530)\end{array}$ & n.d & n.d. & $\begin{array}{r}150 \\
\text { (n.d.-750) }\end{array}$ \\
\hline 3 & $\begin{array}{l}\text { Polycyclic aromatic } \\
\text { hydrocarbons } \\
\left(\Sigma_{16} \mathrm{PAHs}\right)\end{array}$ & $\begin{array}{r}750 \\
(330- \\
2,750) \\
\end{array}$ & $\begin{array}{r}1,22 \\
(850-2,990)\end{array}$ & 60 (n.d.-280) & $\begin{array}{r}350 \\
(n . d .-350)\end{array}$ & $\begin{array}{r}940 \\
(230-5,070)\end{array}$ & $\begin{array}{r}4,17 \\
(340-15,800)\end{array}$ \\
\hline 4 & $\begin{array}{l}\text { Linear } \\
\text { alkylbenzenes } \\
\left(\mathrm{C}_{10}-\mathrm{C}_{13} \text { LABs }\right) \\
\end{array}$ & $\begin{array}{r}7,59 \\
(2,400- \\
50,600)\end{array}$ & $\begin{array}{r}50,21 \\
(12,900- \\
136,700) \\
\end{array}$ & $\begin{array}{r}5,79 \\
\text { (n.d.-28,400) }\end{array}$ & $\begin{array}{r}1,1 \\
(1,000- \\
1,200) \\
\end{array}$ & $\begin{array}{r}32,96 \\
(210- \\
170,000) \\
\end{array}$ & $\begin{array}{r}65,1 \\
(1,400- \\
6,900,000) \\
\end{array}$ \\
\hline 5 & $\begin{array}{l}\text { Di-iso-propyl } \\
\text { naphthalenes } \\
\text { (DIPNs) }\end{array}$ & $\begin{array}{r}990(240- \\
3,700)\end{array}$ & $\begin{array}{r}2,93 \\
(990- \\
21,870)\end{array}$ & $\begin{array}{r}350 \\
(20-14,380)\end{array}$ & $\begin{array}{r}700 \\
(370-1,320)\end{array}$ & $\begin{array}{r}910 \\
(80-32,00)\end{array}$ & $\begin{array}{r}5,13 \\
(240- \\
100,000)\end{array}$ \\
\hline 6 & $\begin{array}{l}\text { Phenylmethoxy- } \\
\text { naphthalene (PMN) }\end{array}$ & $\begin{array}{r}200 \\
\text { (n.d. }-500)\end{array}$ & $\begin{array}{r}1,95 \\
\text { (n.d. - 3,560) }\end{array}$ & $\begin{array}{r}220 \\
(\text { n.d. }-400)\end{array}$ & $\begin{array}{r}270 \\
\text { (n.d. }-270)\end{array}$ & $\begin{array}{r}240 \\
\text { (n.d.-1,600) }\end{array}$ & $\begin{array}{r}740 \\
\text { (n.d. }-3,300)\end{array}$ \\
\hline
\end{tabular}

n.d. : non detectable (LOQ: $0.5 \mathrm{ng}$ ); DDX is sum of DDT, DDE, DDD and DDMU; *) Only single sample was contaminated.

and elimination and biotransformation or detoxification rate in the organism would also contribute in the bioaccumulation of contaminant in aquatic animals (Franke et al., 1994). As filter feeder sedentary species, not only from diffusion of dissolved water, but also green mussel tends to accumulate contaminant from particulate matter, whereas fish accumulate contaminant predominantly from food web (van der Oost et al., 2003).

\subsection{Persistent Organic Pollutants (POPs)}

Persistent organic pollutants (POPs) is defined as organic compounds that resist mainly to photolytic, biological and chemical degradation IPCS (1995). POPs are often halogenated and characterized by low water solubility and in contrast high lipid solubility, leading to their bioaccumulation in fatty tissues. They are group of semi-volatile compounds, enabling them 
to move long distances in the atmosphere before deposition occurs.

DDT metabolites and high molecular weight (HMW) PAHs were among persistent contaminants detected in biological samples and sediment samples from Jakarta Bay. HMW-PAHs consisting of at least four benzene rings are known as persistent pollutants and classified as carcinogenic and mutagenic substances to aquatic biota and human health (ATSDR, 1999). They include benzo[a]pyrene (BaP), benzo[a] antracene $(\mathrm{BaA})$, chrysene $(\mathrm{Chr})$, benzo[b] fluoranthene $(\mathrm{BbF})$, benzo[j]fluoranthene $(\mathrm{BjF})$, benzo[ $k]$ fluoranthene (BkF), dibenzo[a, $h]$ anthracene (DBahA), indeno[1,2,3-cd] pyrene (IP), and benzo[ghi] perylene (BghiP). Figure 3 represents molecular structures of important organic contaminants detected in the present study as they are spatially distributed in different compartments at relatively significant concentration.

Four DDT metabolites were detected in sediment and biological samples of Jakarta Bay, i.e. DDT, DDD, DDE and DDMU. All of these DDT metabolites, furthermore known as DDX, appeared at relatively low concentration in fish sample and river sediment, whereas DDT and DDD were neither detected in green mussel nor in sediment samples. DDE and DDD are the main metabolite products of DDT in the environment, by which DDE is known either more persistent or more toxic than the parent compound DDT (Walker, 1987). Furthermore, microorganism activity, gastrointestinal metabolism, oxygen level or environmental catalyzer (such as $\mathrm{KOH}, \mathrm{NaOH}$ and other strong alkalis) could promote the dechlorination or dehydrochlorination transformation of DDT compounds. Ratio of DDT metabolites over parent compound is often applies to identify their emission behavior (Schwarzbauer et al., 2003). The presence of $p, p^{\prime}$-DDE in sediment and in animal samples in the present study supported that this metabolite is the main degradation product of DDT which is also in line with the earlier investigations (Monirith et al., 2000; Boonyatumanond et al., 2002; Sudaryanto et al., 2005; Bayen et al., 2003).

Comparable DDT metabolites concentrations (DDT, DDE and DDD) in sediment samples from Jakarta Bay were reported earlier by Munawir (2013). Concentrations of DDT metabolites in $P$. viridis in the present study were also comparable to those earlier reported by Monirith et al. (2003) from the same location or from other South East Asian coastal. DDT concentrations in fish in the present study were comparable to those earlier investigation at the same location (Sudaryanto et al., 2007). As presented in Table 3, concentration of DDX in mussels and selected fish in this study was far below the FAO/WHO (2000) maximum residue limit (MRL) of $1,000 \mathrm{ng} / \mathrm{g}$ fat. Similarly, the concentration in sediment was below the threshold of $6,000 \mathrm{ng} / \mathrm{g}$ dw weight (US DI, 1998).

With regard to PAHs contamination, the concentrations in some sediment and mussel samples from Jakarta Bay have exceeded the threshold. A global threshold limit of PAHs in food product has been regulated such as $2 \mathrm{ng} / \mathrm{g} \mathrm{ww}(\mathrm{BaP})$ or equivalent to $12 \mathrm{ng} / \mathrm{g} w \mathrm{w}$ of total $\mathrm{BaP}, \mathrm{BaA}, \mathrm{BbF}$

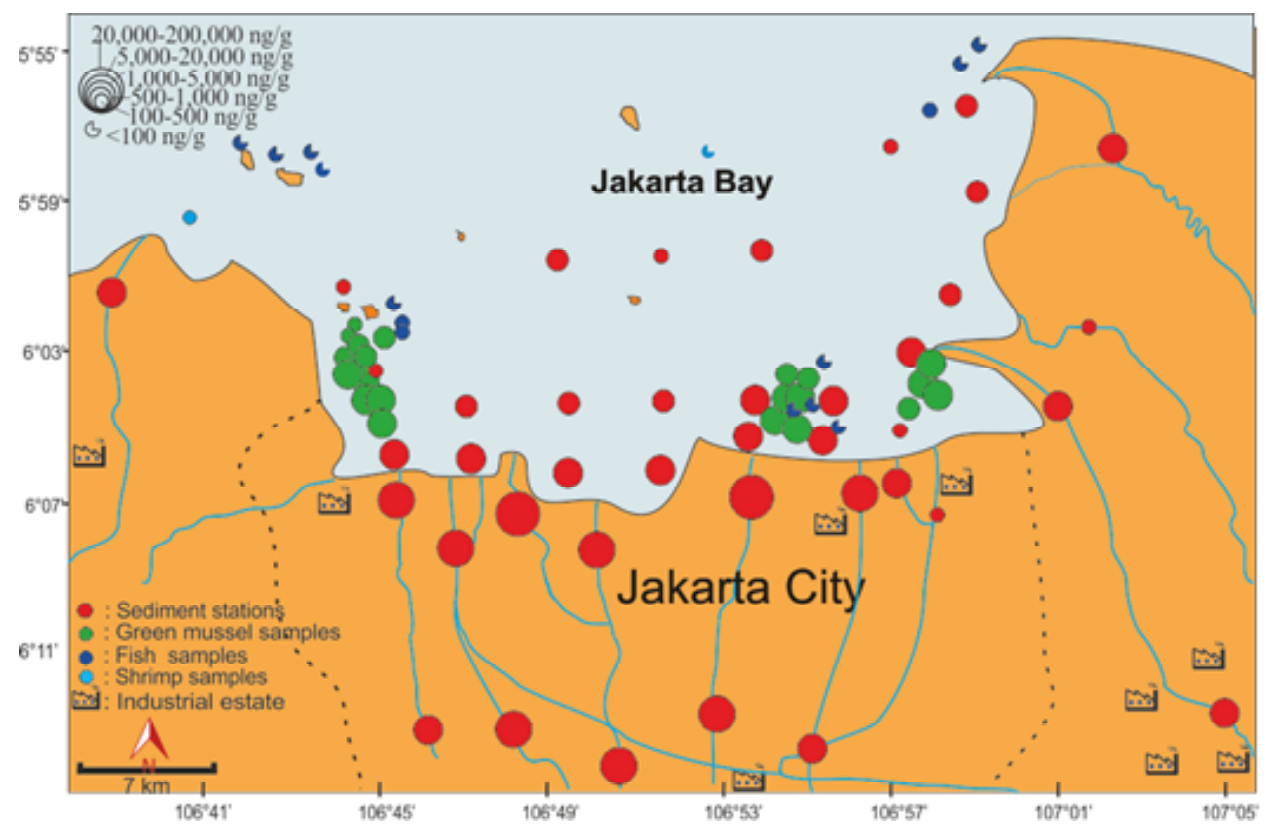

Figure 4. Spatial distribution of PAHs contaminant in Jakarta Bay and JMA rivers sediment (ng/g TOC) and in biota samples (ng/g fat). 
and Chr or $200 \mathrm{ng} / \mathrm{g}$ of total $8 \mathrm{PAHs}$ mentioned previously $(E C, 2006)$. However, those long chains PAHs were not detected in fish and shrimp samples from the study area. The lower bioavailability of high molecular weight (HMW) PAHs could be related to their stronger attachment to sediment particles compared to the low molecular weight PAHs (Baumard et al., 1999). In general, accumulation of PAHs in green mussel was similar to that of sediment samples containing low molecular weight (LMW) and HMW$\mathrm{PAHs}$, indicating ingestion of particulate matter from contaminated waters was predominantly accumulation route into green mussel. In contrast, fish and shrimp predominantly accumulated LMW-PAHs and only partly accumulate pyrene and fluoranthene of HMW supported their bioaccumulation rate was predominantly via aquatic food chain (D'Adamo et al., 1997; Zhao et al., 2014). Incomplete combustion is believed as the main source of PAHs compounds in the environment. Important releases of PAHs include fossil fuel combustion, natural gas for industrial or domestic purposes, forest fire, and the materials used for industrial manufacturing (Maliszewska-Kordybach, 1999; WHO, 2000).

HMW-PAHs have been linked to carcinogenic properties as they could promote active metabolite which can bind to DNA (Neff, 1976). Due to the availability of toxicological data, $\mathrm{BaP}$ has been used as the chemical index to calculate toxicity equivalent values (TEQ) of PAHs contamination. Concentrations of PAHs in Jakarta Bay sediments in this study were the highest so far reported from Asian coastal regions (Takada et al., 1992; Isobe et al., 2004). In the present study, PAH concentrations in mussels were much higher than those reported earlier from the same location or from other South East Asian bays (Tsutsumi et al., 2002, Isobe et al., 2007).

\subsection{Emerging Organic Contaminants}

A number of emerging organic compounds have been detected in sediment and biological samples from Jakarta Bay. The contaminants were dominated by products for technical applications, low molecular weight (LMW) PAHs and halogenated compounds (Table 1). Further on, some contaminants that present in the majority of the samples, potentially harm aquatic environment or human health and possibly applicable as marker for identification of anthropogenic emission were assessed. They include LABs, DIPNs, dichlorobenzene, LMW-PAHs and PMN.

\subsubsection{Linear alkylbenzenes (LABS)}

Linear alkyl benzenes are manufactured in the common surfactant formulation of synthetic detergent, known as linear alkyl benzenes sulfonate/LAS (Eganhouse et al., 1983). These compounds belong to a group of secondary phenyl alkane $\left(\mathrm{C}_{6} \mathrm{H}_{5}-\mathrm{C}_{n} \mathrm{H}_{2 n+1}\right.$, $n=10-14)$. However, as the result of incomplete sulfonation, minor constituent of LABs may remain in the surfactant and are carried over to the detergent. Furthermore, they often appear in municipal and domestic wastes and some would be discharged into riverine system including marine environment. Currently, LABs concentration and profile in sediment, water and aquatic organisms have been widely utilized as molecular tools to assess quality and impact of urban sewage released to the environment (Ishiwatri et al., 1983).

Concentrations of LABs in Jakarta Bay sediments and green mussel samples in the present study was remarkable high and found the highest so far detected in Asian coastal regions (Takada et al., 1992; Isobe et al., 2004). The concentrations in sediment were higher than those from the same location (Rinawati et al., 2012) or from Asian coastal (in Mumbai coast India and in Manila Bay) reported by Isobe et al. (2004). However, no previous reports about LABs concentrations in fish and shrimp species sampled in Indonesian coastal waters or in other Asian regions are available, but the concentration is higher than that in white croaker (Genyonemus lineatus) collected near a large wastewater outfall in southern California (Phillips et al., 2001). So far, Asia Pacific regions being the largest market for LABs and account for $46 \%$ of total market value. LAS plays as the leading application for LABs (97\%), followed by laundry powder production and cleaner application. One of Indonesian company (Unggul Indah Kiat) is among top ten global LABs producers, which are globally dominated by China's companies (Grand View Research, 2015)

\subsubsection{Di-iso-propylnaphthalenes (DIPNs)}

Various industrial applications use DIPNs, such as carbonless paper, while small amount DIPNs are also applied as heat carrier, solvent for pesticides and for other applications (Suzuki et al., 2007). Contamination of DIPN in sediments of Jakarta Bay in the present study, was the highest concentration reported in coastal sediments (Franke et al., 2007; Terasaki et al., 2012). Additionally, DIPNs concentrations in selected fish species from Jakarta Bay were comparable to reported values of fish from Himeji Coast and Shizuoka coastal waters Japan, which concentrations were 1,2 and $9,0 \mathrm{ng} / \mathrm{g}$ respectively (Suzuki et al., 2012; Terasaki et al., 2012). To our knowledge, DIPNs contamination in mussel and shrimp samples has not been reported previously. 


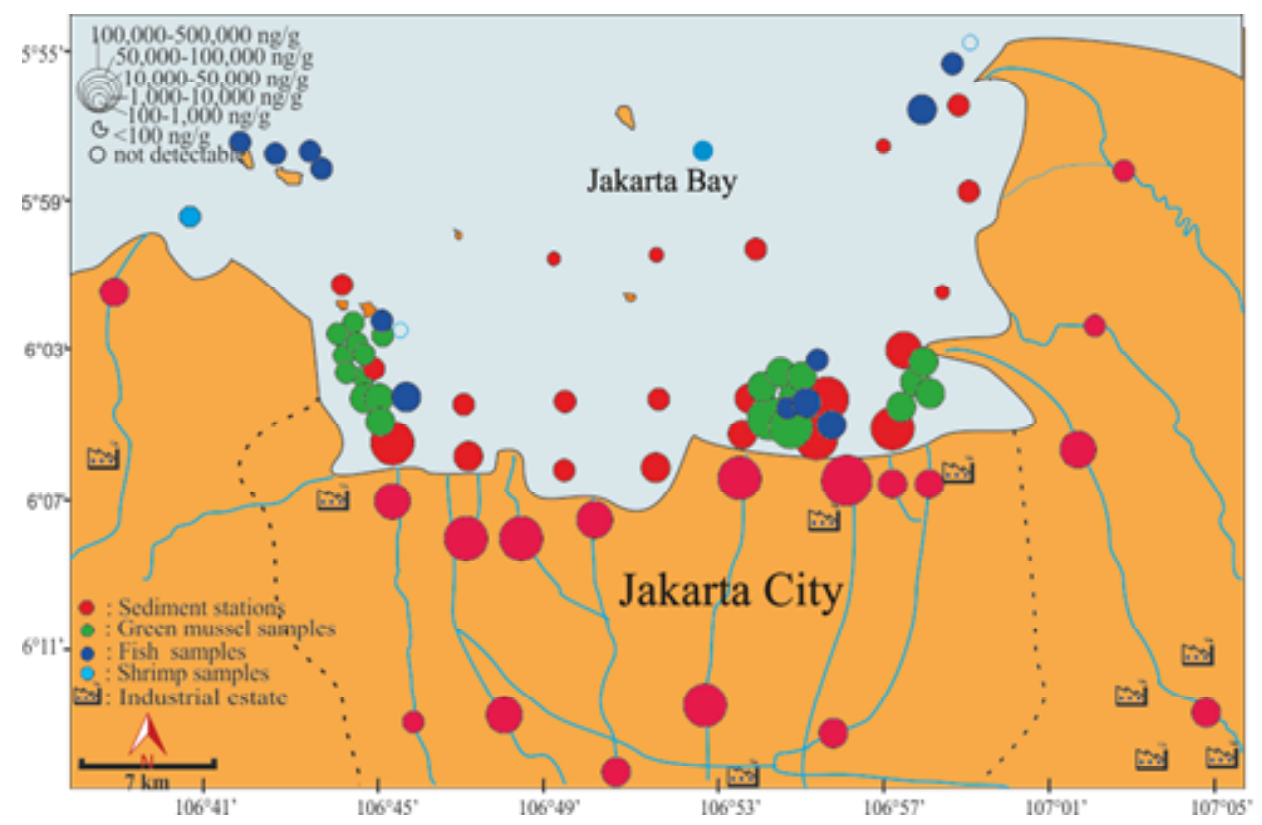

Figure 5. Spatial distribution of LABs contaminant in Jakarta Bay and JMA rivers sediment (ng/g TOC) and in biota samples ( $\mathrm{ng} / \mathrm{g}$ fat).

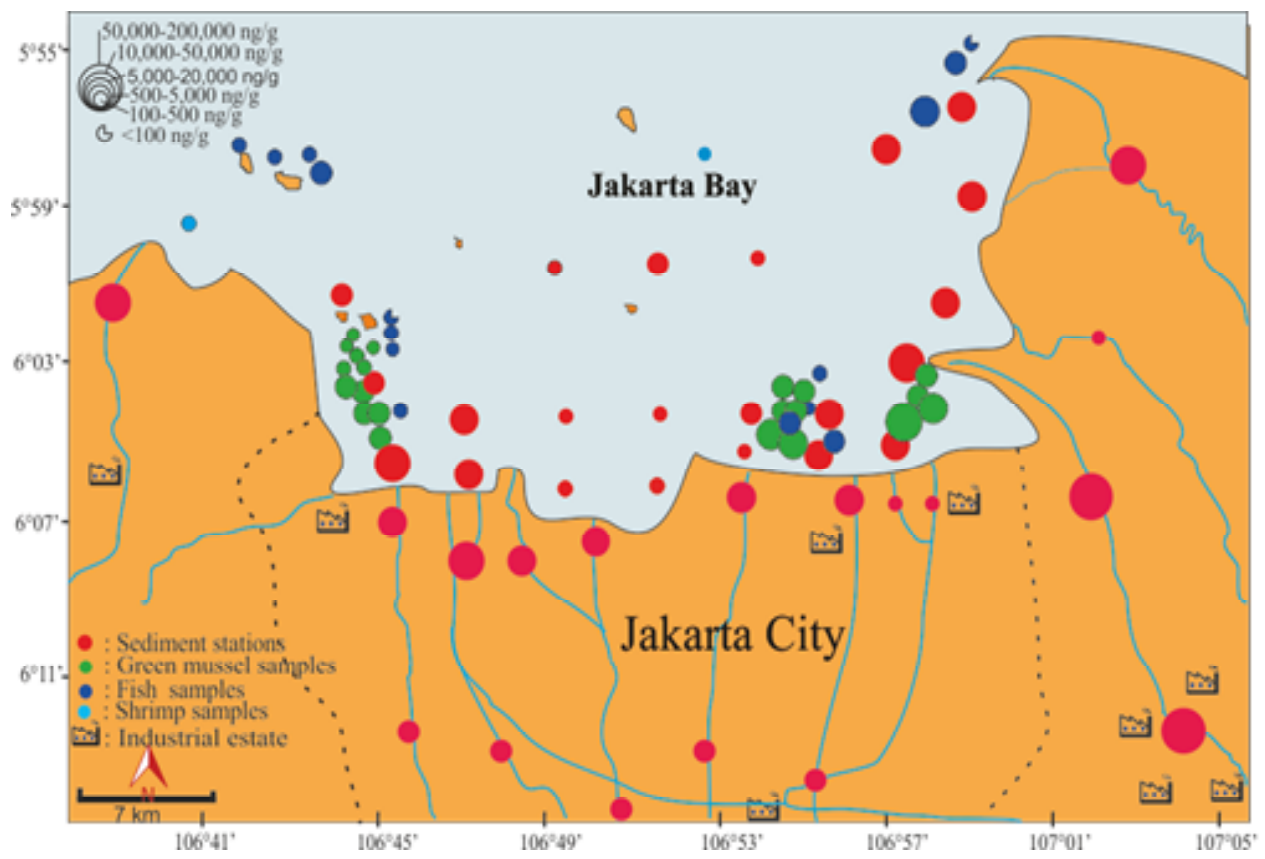

Figure 6. Spatial distribution of DIPNs contaminant in Jakarta Bay and JMA rivers sediment (ng/g TOC) and in biota samples (ng/g fat).

According to Stockholm Convention, DIPNs is classified as not easily biodegradable compound with $\mathrm{T}_{50}$ in water of \pm 2 months. Further toxicity analysis showed $\mathrm{LD}_{50}$ on yellowtail (Seriola quinqueradiata) was approx. $2 \mathrm{~mL} / \mathrm{kg}$ which corresponds to high aquatic toxicity according to the Globally Harmonized System. Long-term toxicity test showed the NOEC study with Daphnia was $13 \mu \mathrm{g} / \mathrm{L}$. Bioassay analysis on rat indicated an increase of liver weight, disturbance of lipid metabolism in the liver and serum and disturbance of glucose metabolism. However no acceptable exposure limits levels are available (UNEP, 2014).

Recently, the Food and Environment Research Agency UK has categorized DIPNs as common contaminants migrating from food contact materials, beside heavy metal, phthalates and polychlorinated 


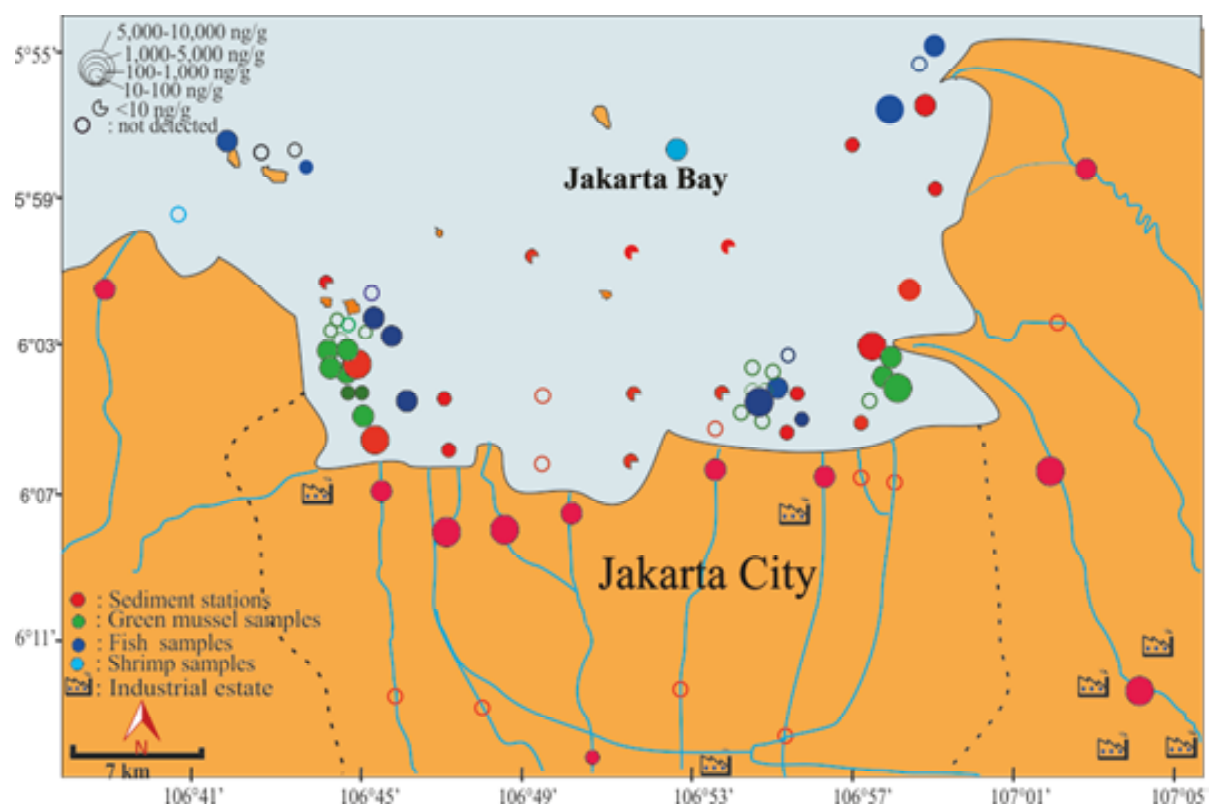

Figure 7. Spatial distribution of PMN contaminant in Jakarta Bay and JMA rivers sediment (ng/g TOC) and in biota samples (ng/g fat).

biphenyl. On a study conducted in the UK detected DIPNs concentration in most samples of retail food packaging at up to $44 \mathrm{mg} / \mathrm{kg}$. Jamnicki et al. (2012) reported that deinking flotation of recycled paper could reduce DIPNs content $50 \%$ from $18.2 \mathrm{ng} / \mathrm{dm}^{2}$ to 9.2 $\mathrm{ng} / \mathrm{dm}^{2}$. Migration simulation test of DIPNs contaminated paper packaging on butter showed migration rate of $74 \%$ compared to initial concentration at $22^{\circ} \mathrm{C}$ (Zülch \& Piringer, 2010).

\subsubsection{Phenylmethoxynaphthalene}

Phenylmethoxynaphthalene (PMN), also known as benzyl naphtyl ether, is classified as irritating substance to the skin and eyes, and as either very toxic to aquatic life with long term effects or may cause long-lasting harmful effects to aquatic life. Acute toxicity test on rainbow trout showed $\mathrm{LD}_{50}$ and NOEL value of $P M N$ was $>0.69 \mathrm{mg} / \mathrm{L}$. Earlier studies showed that the occurrence of PMN in aquatic environments is related to paper industry emissions (Terasaki et al., 2008 \& 2012). Textile manufacturing may also contribute in the emission of PMN contamination into environment (Bridgen et al., 2012), which is used as dye solvent.

In the present study, PMN contamination was detected at relatively similar concentrations in different animal tissue samples at concentrations of 10 to 210 $\mathrm{ng} / \mathrm{g}$ dw in green mussel and from $<10$ to $50 \mathrm{ng} / \mathrm{g} \mathrm{dw}$ in fish samples. PMN concentration in sediments was affected by the season ( $p$-value $<0.05$ ), but this was not observed for animal tissue samples. PMN concentrations in selected fish species in the present study are in line to that reported values for fish samples from Shizuoka coastal waters, which were 3-66 ng/g ww (Terasaki et al., 2012).

\subsubsection{Dichlorobenzenes (DCB)}

Chlorinated benzenes are frequently detected in riverine and estuarine sediment (Kronimus \& Schwarzbauer, 2007). In the present study, DCB were detected in nearly all mussel samples and in some fish species as well as in some river sediment (Table 2). In contrast, DCB was neither appears in coastal sediment nor in shrimp samples. DCBs are either used as product for a large variety of industrial syntheses such as disinfectants, pesticides, deodorizer, air freshener and moth repellent (Baurskens et al., 1994).

The absence of DCB in coastal sediment samples compared to that in green mussel and fish samples suggested this compound is less persistent or more biodegradable in sediment, due to relatively low log $\mathrm{K}_{\text {ow }}$ (3.43-3.63) compared to other compounds (e.g. DIPNs: 6.08). The relatively high concentration of dichlorobenzene in mussel indicated either the higher uptake from dissolve water phase or the lower clearance rate of this compound in mussel rather than in fish species, which was also reported in hexachlorocyclohexane $(\mathrm{HCH})$ by Bute et al. (1991). Noteworthy, identified DCB in riverine sediment suggested their high concentration or relatively close to the point of source. 
Based on the animal study, chlorinated benzenes, especially $1,4-D C B$ is classified as carcinogenic category 3 compound by EU. Threshold value of NOAEL oral for liver cancer in mice was reported as $300 \mathrm{mg} / \mathrm{kg}$ BW per day (EC, 2010). For the aquatic organism, DCB is categorized as very toxic with LC $_{50}$ to fish $1.12-14.2 \mathrm{ppm}$ as well as relatively persistent as the biodegradation rate in surface water and sediment are $4,6 \% /$ day and $0,2 \%$ /day respectively (US EPA, 1998; EC, 2004). This compound is the main formula in the production of air fresheners, moth repellents and toilet block, besides also used in syntheses of pesticides, resins and dyestuff (Euro Chlor, 1999). In general, these products contain more than $95 \%$ of DCB. The use of DCB has been prohibited in Sweden and several federal government of USA (EC, 2010).

\section{Conclusions}

The present study demonstrated the usefulness of a non-target GC/MS screening method for assessing persistent and emerging organic contaminants in aquatic environment and seafood from Jakarta Bay. Most of the identified contaminants have not been reported previously either from the same location or from South East Asia region, such as DIPNs, DCB, DDMU and PMN. Spatial distribution of the organic contaminants showed that eastern part of Jakarta Bay was contaminated more intensively, mainly by industrial emission, compared to that of western part of the bay, which was predominantly contaminated by municipal discharges. This different contaminant intensity was also reflected on the accumulation of the biota, mainly green mussel.

Among the different compartments, sediment accumulated organic contaminants more variety and more concentrated (particularly river sediment), followed by green mussel, fishes and shrimp. Bioavailability of the compounds (such as due to the polarity, persistency and concentration) as well as the behavior of the compartments (uptake and elimination rate) may undergo the different accumulation rate into the different compartments. Mussels for example are known as sedentary filter feeder that accumulates contaminant mainly from soluble fraction or particulate matters whereas fish predominantly accumulates contaminants from food web through bio-magnification.

Since lipophilic organic compounds are typically accumulated in lipid containing tissue of the animal, it would be worthy to investigate contaminant concentration in different fatty organs. Investigation of low volatilized polar compounds using liquid chromatographic approach is also important to get the whole overview of the organic contaminants.

\section{Acknowledgements}

We thank the SPICE-III Project (Science for the Protection of Indonesian Coastal Ecosystems) funded by the German Federal Ministry of Education and Research (BMBF, Grant No.03F0641E) for the research funding and DAAD (Germany Academic Exchange Service) for a scholarship provided for the main author. Colleagues from BBP4BKP (Jakarta) and Ms. Yvonne Esser (RWTH Aachen University) are thanked for their support during the sampling campaign and laboratory analyses.

\section{References}

Anonymous. (2007). Teluk Jakarta, 80 Persen Pencemaran oleh Sampah dari Darat (The Jakarta Bay, 80 percent of contaminant delivered from terestrial waste).http://www.kompas.com/kompascetak/0706/02/metro/ 3570312.htm

AOAC. (2002). Official methods of analysis (996.01) Fat (total, saturated, unsaturated, and unsaturated) in food products (17th ed.). Association of Official Analytical Chemists. USA.

ATSDR. (1999). Toxycological Profile of Polycyclic Aromatic Hydrocarbons. Agency for toxic substances and desease registry. US Department of Health and Human Services. Washington.

Baumard, P., Budzinski, H., Garrigues, P., Narbonne, J.F., Burgeot, T., Michel, X., \& Bellocq, J. (1999). Polycyclic aromatic hydrocarbon (PAH) burden of mussels (Mytilus sp.) in different marine environments in relation with sediment $\mathrm{PAH}$ contamination, and bioavailability. Mar Environ Res, 47(5): 415-439.

Baurskens, J.E.M., Dekker, C.G.C., van den Heuvel, H., Swart, M. \& de Wolf, J. (1994). Dechlorination of chlorinated benzenes by an anaerobic microbial consortium that selectively mediates the thermodynamic most favorable reactions. Environ. Sci. Technol. 28: 701-706.

Bayen, S., Thomas, G.O., Lee, H.K. \& Obbard, J.P. (2003). Occurrence of PCBs and PBDEs in green mussels (Perna viridis) from Singapore, Southeast Asia. Environ. Toxicol. Chem. 10: 2432-2437.

Boonyatumanond, R., Jaksakul, A., Puncharoen, P., \& Tabucanon, M.S. (2002). Monitoring of organochlorine pesticides residues in green mussels (Perna viridis) from the coastal area of Thailand. Environ. Pollut. 119: 245-252.

BPLHD. (2012). Environmental status of Jakarta Province. Annual Report 2012. Badan Pengelola Lingkungan Hidup. DKI Jakarta.

BPS. (2014). Jakarta dalam Angka. Local Government of Jakarta Province. Local Government of Jakarta Province. Central Agency of Statistic.

Brigden, K., Labunska, I., House, E., Santillo, D. \& Johnston, P. (2012). Hazardous chemicals in branded textile products on sale in 27 places during 2012. Greenpeace Research Laboratories Technical Report 06/2012. 
D’adamo, R., Pelosi, S., Trotta, P., \& Sansone, G. (1997). Bioaccumulation and biomagnification of polycyclic aromatic hydrocarbons in aquatic organisms. Mar. Chem. 56(1): 45-49.

Díaz, R., lbáñez, M., Sancho, J.V., Hernández, F. (2012). Target and non-target screening strategies for organic contaminants, residues and illicit substances in food, environmental and human biological samples by UHPLC-QTOF-MS. Anal. Methods. 4:196-209.

Dsikowitzky, L., Nordhaus, I., Jennerjahn, T., Khrycheva, P., Sivatharshan, Y., Yuwono,E. \& Schwarzbauer, J. (2011). Anthropogenic organic contaminants in water, sediments and benthic organisms of the mangrovefringed Segara Anakan Lagoon, Java, Indonesia. Mar. Pollut. Bull. 62: 851-862.

Dsikowitzky, L., Dwiyitno, Heruwati, E., Ariyani, F., Irianto, H. E. \& Schwarzbauer, J. (2014). Exceptionally high concentrations of the insect repellent $\mathrm{N}, \mathrm{N}$-diethyl$\mathrm{m}$-toluamide (DEET) in surface waters from Jakarta, Indonesia. Environ. Chem. Lett. 12(3), 407-411.

Dsikowitzky, L., Sträter, M., Dwiyitno, Ariyani, F., Irianto, H.E., \& Schwarzbauer, J. (in draft). First comprehensive screening of lipophilic organic contaminants in surface waters of the megacity Jakarta, Indonesia. Special Issue, Mar. Pollut. Bull. http://www.sciencedirect.com/science/article/pii/ S0025326X16300777.

Dwiyitno, Dsikowitzky, L., Nordhaus, I., Andarwulan, N., Irianto, H.E., Lioe, H.N., Ariyani, F., Kleinertz, S. \& Schwarzbauer, J. (in draft). Accumulation pattern of lipophilic organic contaminants in economic important mussel and fish species from Jakarta Bay, Indonesia. Marine Pollut. Bull. http:// www.sciencedirect.com/science/article/pii/ S0025326X16300339.

EC. (2004). 1,4-Dichlorobenzene, CAS No: 106-46-7, EINECS No: 203-400-5, Risk Assessment Final Report, European Commission. http:// ecb.jrc.ec.europa.eu/documents/ExistingChemicals/risk_assessment/report/14dichloro benzenereport001.pdf (accessed on 10 October 2015).

EC. (2006). Commission regulation No1881/2006. Setting maximum levels for certain contaminants in food stuffs. European Commission. Official J. Europ. Union. L364/5-24.

EC. (2010). Socio-Economic Evaluation arising from a Proposal for Risk Reduction Measures related to Restrictions on 1,4 Dichlorobenzene. DirectorateGeneral Enterprise and Industry. European Commission.

Eganhouse, R.P., Blumfield, D.L., Kaplan, I.R. (1983). Long-chain alkylbenzens as molecular tracer of domestic wastes in the marine environment. Environ. Sci. Technol. 17:523-530.

Euro Chlor. (1999). 1,4-dichlorobenzene. Euro Chlor Risk Assessment for The Marine Environment. http:// www. eurochlor.org/media/49301/8-11-410_marine_ra_14-dichlorobenzene.pdf.
FAO/WHO. (2000). Pesticide residue in food and feed. Codex Alimentarius-International Food Standard.

Fang, J., Wu K., Zheng, G.J., Au., D.W., Lam, P.K., \& Shin, P.K. (2009). The use of muscle burden in rabbit fish Siganus oramin for monitoring polycyclic aromatic hydrocarbons and polychlorinated biphenyls in Victoria Harbour, Hong Kong and potential human health risk. Sci. Total. Environ. 407(14):4327-4332. doi: 10.1016/j.scitotenv.2009.03.033.

Franke, S., Grunenberg, J., \& Schwarzbauer, J. (2007). The isomer-specific analysis of diisoprophyl naphthalenes. Intern. J. Environ. Chem. 87(6):437448.

Franke, C., Studinger, G., Berger, G., Bohling, S., Bruckman, U., Cohors-Fresenborg, D., Johncke, U. (1994). The Assesment of Bioaccumulation. Chemosphere. 29: 1501-1514.

Grand View Research. (2014). Global Linear Alkyl Benzene (LAB) Market Analysis By Application (Heavy Duty Laundry Liquids, Laundry Powders, Light Duty Dish Washing Liquids, Industrial Cleaners, Household Cleaners) Expected to Reach USD 9.27 Billion by 2020: Grand View Research, Inc http:// www.grandviewresearch.com/. Accesed on 22 September 2015.

Grigoriadou, A., Schwarzbauerm, J., \& Georgakopoulos, A. (2008). Molecular indicators for pollution source identification in marine and terrestrial water of the industrial area of Kavala city, North Greece. Environ. Pollut. 151: 231-242

Grilo, T.F., Cardoso, P.G., Pato, P., Duarte, A.C., \& Pardal, M.A. (2014). Uptake and depuration of PCB-153 in edible shrimp Palaemonetes varians and human health risk assessment. Ecotoxicol. Environ. Safety. 101: 97-102

Heiri, O., Lotter, A. F., \& Lemcke, G. (2001). Loss on ignition as a method for estimating organic and carbonate content in sediments: reproducibility and comparability of results. J. Paleolimnol. 25(1), 101110.

Hernández, F., Portolés, T., Pitarch, E., \& López, F.J. (2007). Target and Non-target Screening of Organic Micro-pollutants in Water by Solid-Phase Microextraction Combined with Gas Chromatography/HighResolution Time-of-Flight Mass Spectrometry. Anal. Chem.79 (24): 9494-9504.

Hernández, F., Sancho, J., Ibáñez, M., Abad, E., Portolés, T., \& Mattioli, L. (2012). Current use of high-resolution mass spectrometry in the environmental sciences. Anal. Bioanal. Chem. 403(5):1251-64. doi: 10.1007/ s00216-012-5844-7.

Hird, S.J., Lau, B.P.Y., Schumacher, R., \& Krska, R. (2014). Liquid chromatography-mass spectrometry for the determination of chemical contaminants in food. TrAC Trends Analytic. Chem. 59:59-72. doi:10.1016/ j.trac.2014.04.005.

Ibanez, M., Sancho, J.V., McMillan, D., Rao, R., \& Hernandez, F. (2008). "Rapid non-target screening of organic pollutants in water by ultraperformance liquid chromatography couple to time-of-flight mass 
spectrometry." Trends Analytic. Chem. 27(5): 481489.

IPCS. (1995). An Assessment Report on: DDT,AldrinDieldrin-Endrin-Chlordane, Heptachlor-Hexa chlorobenzene, Mirex-Toxaphene, Polychlorinated Biphenyls, Dioxins, and Furans. The International Programme on Chemical Safety (IPCS) within the framework of the Inter-Organization Programme for the Sound Management of Chemicals (IOMC). http:/ /www.chem.unep.ch/pops/indxhtms/asses0.html.

Ishiwatari, R., Takada, H., Yun, S.J., \& Matsumoto, E. (1983). Alkylbenzen pollution of Tokyo Bay sediments. Nature. 301: 599-600.

Isobe, K.O., Zakaria, M.P., Chiem, N.H., Minh, L.Y., Prudente, M., Boonyatumanond, R., Saha, M., Sarkar, S., \& Takada, H. (2004). Distribution of linear alkylbenzenes (LABs) in riverine and coastal environments in South and Southeast Asia. Water Res. 38(9): 2449-2459.

Isobe, T., Takada, H., Kanai, M., Tsutsumi, S., Isobe, K.O., Boonyatumanond, R., \& Zakaria, M.P. (2007). Distribution of polycyclic aromatic hydrocarbons (PAHs) and phenolic endocrine disrupting chemicals in South and Southeast Asian mussels. Environ. Monit. Assess. 135:423-440.

Jamnicki, S., Lozo, B., Rutar, V., \& Barusic, L. (2012). The suitability of using recycled paper as a direct food packaging. The $1^{\text {st }}$ Conference BioMatPack: Sustainable and Renewable Packaging Industrial Opportunities. Eds. E. Bobu, P. Obrocea. Fundacion Itene. Barcelona 17 May 2011.

Kim, K.S., Hong, K.H., Ko, Y.H., Yoon, K.D. \& Kim, M.G. (2003). Emission characteristics of PCDD/Fs in Diesel Engine with Variable Load Rate. Chemosphere. 53: 601-607.

Kronimus, A. \& Schwarzbauer, J. (2007). Non-target screening of extractable and non-extractable organic xenobiotics in riverine sediments of Ems and Mulde Rivers, Germany. Environ. Res. 147(1):176-186. doi:10.1016/j.envpol.2006. 08.014.

Maliszewska-Kordybach, B. (1999). Sources, Concentrations, Fate and Effects of Polycyclic Aromatic Hydrocarbons (PAHs) in the Environment. Part A: PAHs in Air. Polish J. Environ. Studies. 8(3): 131-136.

Monirith, I., Nakata, H., Watanabe, M., Takahasi, S., Tanabe, S., Tana, T.S. (2000). Organochlorine contamination in fish \& mussels from Cambodia \& Other Asian Countries. Water Sci. Tech. 42(7-8): 241252.

Monirith, I.D., Ueno, S., Takahashi, H., Nakata, A., Sudaryanto, A., Subramanian, N., Karuppiah, S., Ismail, A., Muchtar, M., Zheng, J., Richardson, B.J, Prudente, M., Hue, N.D., Tana, T.S, Tkalin, A.V. \& Tanabe, S. (2003). Asia-Pacific mussel watch: monitoring contamination of persistent organochlorine compounds in coastal waters of Asian countries. Mar. Pollut. Bull. 46: 281-300.

Munawir, K. (2013). Differences Concentration Distribution and Source of in Organochlorine
Pesticides in Water and Sediment in Jakarta Bay Waters during Transition Season. Trop. J. Mar. Sci. 5(1):122-130.

Neff, J.M., Cox, B.A., Dixit, D., \& Anderson, J.W. (1976). Accumulation and release of petroleum-derived aromatic hydrocarbons by four species of marine animals. Mar. Biol. 38(3), 279-289.

Nkpaa, K.W., Bassey, E.E. \& Wegwu, M.O. (2013). Evaluation of Polycyclic Aromatic Hydrocarbon (PAH) Concentrations in Crabs and Shrimps from Crude Oil Polluted Waters of Ogoniland in Rivers State , Nigeria. IOSR J. Environ. Sci. Toxicol. Food. Technol. 4 (6): 73-80.

Peterman, P.H, \& Delfino, J.J. (1990). Identification of Isoprophylbiphenyl, Alkyl Dipehnylmethanes, Diisoprophylnaphthalene, Linear Alkyl Benzenes and Other Polychlorinated Biphenyl Replacement Compounds in Efluents, Sediments and Fish in the Fox River System, Wisconsin. Biomed. Environ. Mass Spectrom. 19: 755-770.

Phillips CR, Venkatesan MI, Lin T. (2001). Linear alkylbenzenes in muscle tissues of white croaker near a large ocean outfall in southern California, USA. Environ. Toxicol. Chem. 20(2):231-2388.

Pierard, C., Budzinski, H. \& Garrigues, P. (1996). Grainsize distribution of polychlorobiphenyls in coastal sediments. Environ. Sci. Technol. 31: 2686-2691.

Ricking, M. \& Schwarzbeur, J. (2012). DDT Isomers and Metabolites in the Environment, An overview. Environ. Chem. Lett. 10:317-323.

Rinawati, Koike, T., Koike, H., Kurumisawa, R., Ito, M., Sakurai, S., Togo, A., Saha, M., Arifin, Z. \& Takada, H. (2012). Distribution, source identification, and historical trends of organic micropollutants in coastal sediment in Jakarta Bay, Indonesia. J. Hazard Mat. 217-218: 208-216.

Schwarzbauer, J., Littke, R. \& Weigelt, V. (2000). Identification of specific organic contaminants for estimating the contribution of the Elbe river to the pollution of the German Bight. Org. Geochem. 31: 1713-1731.

Schwarzbauer, J., Ricking, M. \& Littke, R. (2003). DDTrelated compounds bound to the non-extractable particulate matter in sediments of the Teltow Canal, Germany. Environ. Sci. Technol. 37,488-495.

Schwarzbauer, J. \& Ricking, M. (2010). Non-target screening analysis of river wateras compound related base for monitoring measures. Environ. Sci. Pollut. Res.17: 934-947. doi:10.1007/s11356-009-0269-3.

Schymanski, E.L., Singer, H.P., Longrée, P., Loos, M., Ruff, M., Stravs, M.A., Vidal, C.R. \& Hollender, J. (2014). Strategies to Characterize Polar Organic Contamination in Wastewater: Exploring the Capability of High Resolution Mass Spectrometry. Environ. Sci. Technol., 2014, 48 (3), pp 1811-1818. DOI: $10.1021 /$ es4044374.

Shaikh, H., Memon, N., Bhanger, M.I. \& Nizamani, S.M. (2014). GC/MS Based Non-target Screening of Organic Contaminants in River Indus and its 
Tributaries in Sindh (Pakistan). Pak. J. Anal. Environ. Chem. 15(1):42-65

Sudaryanto, A., Kunisue, T., Tanabe, S., Niida, M. \& Hashim, H. (2005). Persistent organochlorine compounds in human breast milk from mothers living in Penang and Kedah, Malaysia. Arc. Environ. Contam. Toxicol. 49: 429-437.

Sudaryanto, A., Monirith, I., Kajiwara, N., Takahashi, S., Hartono, P., Muawanah, Omori, K., Takeoka, H. \& Tanabe, S. (2007). Levels and distribution of organochlorines in fish from Indonesia. Environ. Int. 33: 750-758.

Suzuki, M., Matsumura, C., Moriguchi, Y. \& Nakano, T. (2007). Investigation of monoisoprophylnaphthalene, di-isoprophylnaphthalene and tri-isoprophylnaphthalene in the environment around the paper recycling plant. Organohalogen Comp. 69:2910-2913.

Suzuki, M., Matsumura, C., Nakano, T., \& Imaishi, H. (2012). Investigation of environmental contamination of mono-isoprophylnaphthalene, diisoprophylnaphthalene and triisoprophylnaphthalenein Hyogo Japan. Environ. Sci. Pollut. Res. 19(9):3959-68. doi: 10.1007/s11356-0120987-9

Takada, H., Ishiwatari, R. \& Ogura, N. (1992). Distribution of Linear Alkylbenzenes (LABs) and Linear alkylbenzene-sulfonates (LAS) in Tokyo Bay sediments. Estuar. Coast. Self. Sci. 35: 141-156.

Takarina, N.D. \& Adiwibowo, A. (2011). Impact of heavy metals contamination on the biodiversity of marine benthic organisms in Jakarta Bay. J. Coast. Develop. 14(2): 168-171.

Terasaki, M., Fukazawa, H., Tani, Y. \& Makino, M. (2008). Organic pollutants in paper-recycling process water discharge areas: First detection and emission in aquatic environment. Environ. Pollut. 151:53-59.

Terasaki, M., Jozuka, K., \& Makino, M. (2012). Identification and accumulation of aromatic sensitizers in fish from paper recycling in Japan. Environ. Toxicol. Chem. 31(6): 1202-1208. doi: 10.1002/etc. 1812

Tsutsumi, S,. Yamaguchi, Y., Nishida, I., Akiyama, K., Zakaria, M.F. \& Takada, H. (2002). Alkylbenzenes in mussels from south east Asian Coast as a molecular tool to assess sewage impact. Marine Pollut. Bull. 45:324-331.

UNEP. (2014). Technical Work: Process for the Evaluation of Perfluorooctane Sulfonic acid, its Salts and Perfluorooctane Sulfonyl Fluoride Pursuant to paragraphs 5 and 6 of part III of Annex B to the Stockholm Convention on Persistent Organic
Pollutants. Persistent Organic Pollutants Review Committee Tenth meeting Rome, 27-30 October 2014. UNEP/POPS/POPRC.10/INF/9.

Uneputty, P.A, \& Evanst, S.M. (1997). Accumulation of Beach Litter on Islands of the Pulau Seribu Archipelago, Indonesia. Mar. Pollut. Bull. 34(8):652655.

US DI. (1998). Guidelines for Interpretation of the Biological Effect of Selected Constituentsin Biota, Water, and Sediment: DDT. Bureau of Reclamation, U.S. Fish and Wildlife Service, U.S. Geological Survey, Bureau of Indian Affairs. United States Department of the Interior.

US EPA. (1998). National Volatile Organic Compound Emission Standards for Consumer Products, Federal Register: September 11, 1998 (Vol.63, Nr. 176), US Environmental Protection Agency. http://www.epa.gov/ EPA-AIR/1998/September/Day-11/a22660.htm (accessed on 22 October 2015).

Van der Oost, Beyer, D.J. \& Vermeulen, N.P.E. (2003). Fish Bioaccumulation and Biomarkers in Environmental Risk Assessment/ : A Review. Environ. Toxicol. Pharmacol, 13: 57-149.

Walker, C.H. (1987). Organic Pollutants: an Ecotoxicological Perspective. Taylor and Francis Group, LLC. CRC Press, 434p.

WHO. (2000). Air Quality Guidelines-2 ${ }^{\text {nd }}$ Ed.. Chapter 5.9: Polycyclic aromatic hydrocarbon. (PAHs) WHO Regional Office for Europe, Copenhagen, Denmark, 2000. http://www.euro.who.int/_data/assets/pdf_file/ 0015/123063/AQG2ndEd_5_9PAH.pdf

Williams, T.M., Rees, J.G., \& Setiapermana, D. (2000). Metals and trace organic compounds in sediments and waters of Jakarta Bay and the Pulau Seribu complex, Indonesia. Mar. Pollut. Bull. 40: 277-285.

Zhao, Z., Zhang, L., Cai, Y., \& Chen, Y. (2014). Distribution of polycyclic aromatic hydrocarbon (PAH) residues in several tissues of edible fishes from the largest freshwater lake in China, Poyang Lake, and associated human health risk assessment. Ecotoxicol. Environ. Safety, 104, 323-331.

Zou, Y.Y. \& Schreiber, A. (2012). Quantitation and Identification of Organotin Compounds in Food, Water, and Textiles Using LC-MS/MS. Food \& Environ. Publ. Nr: 6690212-01, AB SCIEX Concord, Ontario (Canada). http://sciex.com/Documents/brochures/ Organotin_pesticides_QTRAP4k_6690212.pdf

Zülch, A. \& Piringer, O. (2010). Measurement and modelling of migration from paper and board into foodstuffs and dry food simulants. Food Additives Contam. 27(9): 1306-1324. 